\title{
Modeling and numerical study of actuator and sensor effects for a laminated piezoelectric plate
}

\author{
L. Costa ${ }^{\text {a }}$, I. Figueiredo ${ }^{\text {b,* }}$, R. Leal ${ }^{\text {c }}$, P. Oliveira ${ }^{\text {a }}$, G. Stadler $^{b}$ \\ a Departamento de Produção e Sistemas, Escola de Engenharia, Universidade do Minho, Campus de Azurém, 4800-058 Guimarães, Portugal \\ ${ }^{\mathrm{b}}$ Departamento de Matemática, Faculdade de Ciências e Tecnologia, Universidade de Coimbra, Apartado 3008, 3001-454 Coimbra, Portugal \\ ${ }^{\mathrm{c}}$ Departamento de Engenharia Mecânica, Universidade de Coimbra, Pinhal de Marrocos, 3001-201 Coimbra, Portugal
}

Received 19 May 2006; accepted 15 November 2006

Available online 26 January 2007

\begin{abstract}
The aim of this paper is to derive a reduced model for a piezoelectric plate and to study its actuator and sensor capabilities. In the first part, we focus on the asymptotic modeling for thin plates formed by stacking layers of different piezoelectric materials. In the asymptotic model, the mechanical and electric unknowns are shown to be partly decoupled. In the second part, we study the actuator and sensor capabilities of this model. We use two discrete non-differentiable multi-objective optimization problems, which are solved by genetic algorithms. Several numerical results are reported.
\end{abstract}

(c) 2006 Elsevier Ltd. All rights reserved.

Keywords: Piezoelectric material; Laminated plate; Actuator; Sensor; Genetic algorithms

\section{Introduction}

Piezoelectric materials belong to a class of smart materials that exhibit electromechanical coupling, which provides them with actuator and sensor capabilities. The mechanical deformation generated by the application of an external electric field to the material is known as piezoelectric effect, and the converse phenomenon as sensor effect. These properties make piezoelectric devices extremely useful in a wide range of practical applications in aerospace, mechanical, electrical, civil and biomedical engineering (see, e.g., $[1,2])$.

The aim of this paper is twofold: In the first part, we derive a new asymptotic model for a thin laminated plate formed by stacking several layers of different piezoelectric materials. Then, in the second part, the actuator and sensor capabilities of this plate model are studied.

The first part (Section 2), in which the plate model is derived, is composed of three subsections. After the nota-

\footnotetext{
Corresponding author. Tel.: +351 239791 150; fax: +351 239832568.

E-mail address: isabelf@mat.uc.pt (I. Figueiredo).

$U R L:$ http://www.mat.uc.pt/ isabelf (I. Figueiredo).
}

tions and the description of the three-dimensional plate equations (Section 2.1), in Section 2.3 we state the twodimensional (2D) asymptotic model for the thin laminated plate. This is accomplished in two steps. Firstly, in Section 2.2 the plate model found in [11] (for a thin piezoelectric plate with monoclinic elastic coefficients and modified piezoelectric coefficients independent of the thickness variable) is generalized to the case of a completely nonhomogeneous anisotropic piezoelectric plate (cf. (7), Theorem 2.1 and Corollary 2.1). It is found that the solution of this 2D asymptotic model (given by (12)-(18) consists of the Kirchhoff-Love mechanical displacement (whose tangential and transverse components are coupled) and the electric potential; the latter is an explicit function of the difference of the prescribed electric potentials on the lower and upper face of the plate, and of the tangential and transverse mechanical displacements of the plate's middle plane (cf. (12) in Theorem 2.1). This is in contrast to the models in $[9,11]$, where the transverse and tangential components of the mechanical displacement are decoupled and a different formula for the electric potential is found. In Section 2.3, our 2D-model (12)-(18) for non-homogeneous 
anisotropic piezoelectric plates is specified to the case of a thin laminated plate formed by stacking several layers of different piezoelectric anisotropic materials. Clearly, this type of laminated plate can be considered as a plate made of one lamina with a non-homogeneous anisotropic material. Assuming that the material coefficients of each layer are independent of $x_{3}$ (the middle plane of the plate is assumed to lie in the $x_{1} x_{2}$-plane), we prove that the electric potential is a quadratic polynomial in $x_{3}$ with coefficients that depend on the tangential and transversal mechanical displacements of the plate's middle plane (cf. (32)). This means, that the electric potential can be easily derived a posteriori from the mechanical deformation.

In the second part of the paper (Section 3), we numerically study the actuator and sensor capabilities of our model. Section 3.1 is devoted to a brief description of the finite element model for the 2D asymptotic laminated plate. This discrete model is obtained applying standard finite elements to the 2D asymptotic laminated plate model found in Section 2.3. Using two multi-objective and non-differentiable optimization problems, in Section 3.2 we study the smart capabilities of a laminated plate. For the actuator effect, we intend to maximize the displacement while minimizing the number of regions where a non-zero electric potential is applied. A similar multi-objective problem is used to study the sensor effect. These optimization problems are solved by the elitist genetic algorithms described in [3]. We remark that the numerical study for the actuator problem presented in this paper constitutes a continuation of results in the previous work [4]. Differently from the model considered in the present paper, in [4] the plates under consideration are mechanically monoclinic and the modified piezoelectric coefficients are independent of the thickness variable. Thus, the tangential and transversal mechanical displacements are uncoupled and the problem simplifies considerably.

Finally, in Section 3.3 several numerical tests are reported. They illustrate the actuator and the sensor capabilities of a thin laminated plate formed by two piezoelectric anisotropic layers made of PZT materials.

Before finishing this introduction, we mention related approaches and results that can be found in literature. We first refer to $[5,6]$ for the description of the asymptotic method in the case of thin elastic plates. Extensions of this method can be found in [7-11] for thin piezoelectric plates and in [12] for thin piezoelectric shells. For the case of piezoelectric plates, the corresponding asymptotic models differ mainly due to the different materials, scaling techniques and electric boundary conditions. We refer as well the works [13-15] for the modeling (without using the asymptotic method), the finite element discretisation and the numerical simulation of the actuator effect of piezoelectric thin shells. Theoretical formulations for the analysis of laminated composite plates with integrated sensors and actuators are presented in [16]. Analysis, modeling and numerical simulation of piezoelectric actuators can be found in [17-19]. For other works reporting the optimal placement of piezoelectric actuators and sensors in plates we refer, e.g., $[20,21]$ and the for the same kind of problem using genetic algorithms see, e.g., [22].

\section{The asymptotic model}

In this section, we first describe in Section 2.1 our notations and recall the three-dimensional (3D) equations for a thin non-homogeneous anisotropic piezoelectric plate. Then, in Section 2.2 we give in (7) the variational formulation of the corresponding two-dimensional (2D) asymptotic model. Moreover, in Theorem 2.1 we prove that this variational formulation is equivalent to a simpler one, and, in Corollary 2.1 we show that Theorem 2.1 generalizes theorem 3.4 of [11]. In Section 2.3, the 2D asymptotic plate model defined in Theorem 2.1 is considered for the special case of a thin laminated plate made of stacked layers of different piezoelectric anisotropic materials.

\subsection{The $3 D$ piezoelectric plate model}

Let $O X_{1} X_{2} X_{3}$ be a fixed three-dimensional coordinate system, $\omega \subset \mathbb{R}^{2}$ a bounded domain with a Lipschitz continuous boundary $\partial \omega$, and $\gamma_{0}, \gamma_{1}, \gamma_{e}$ and $\gamma_{s}$ subsets of $\partial \omega$. We assume that $\gamma_{0} \neq \emptyset$ and use $\gamma_{1}=\partial \omega \backslash \gamma_{0}$ and $\gamma_{s}=\partial \omega \backslash \gamma_{e}$, where $\gamma_{e}$ can be empty. We consider the sets

$$
\begin{aligned}
& \Omega=\omega \times(-h, h), \quad \Gamma_{ \pm}=\omega \times\{ \pm h\}, \quad \Gamma_{+}=\omega \times\{+h\}, \\
& \Gamma_{-}=\omega \times\{-h\}, \quad \Gamma_{D}=\gamma_{0} \times(-h, h), \quad \Gamma_{1}=\gamma_{1} \times(-h, h), \\
& \Gamma_{N}=\Gamma_{1} \bigcup \Gamma_{ \pm}, \quad \Gamma_{e N}=\gamma_{s} \times(-h, h), \quad \Gamma_{e D}=\Gamma_{ \pm} \bigcup\left(\gamma_{e} \times(-h, h)\right),
\end{aligned}
$$

where $\bar{\Omega}=\bar{\omega} \times[-h, h]$ (that is, $\Omega$ and its boundary) represents a thin plate with middle surface $\omega$ and thickness $2 h$, with $h>0$ a small constant, $\Gamma_{+}$and $\Gamma_{-}$are, respectively, the upper and lower faces of $\Omega$, the sets $\Gamma_{D}, \Gamma_{1}$ and $\Gamma_{e N}$ are portions of the lateral surface $\partial \omega \times(-h, h)$ of $\Omega$, and finally $\Gamma_{N}$ and $\Gamma_{e D}$ are portions of the boundary $\partial \Omega$ of $\Omega$. The points of $\Omega$ are denoted by $x=\left(x_{1}, x_{2}, x_{3}\right)$, where the first two components $\left(x_{1}, x_{2}\right) \in \omega$ and $x_{3} \in(-h, h)$.

Throughout the paper, the Latin indices $i, j, k, l, \ldots$ belong to the set $\{1,2,3\}$, the Greek indices $\alpha, \beta, \mu, \ldots$ vary in the set $\{1,2\}$ and the summation convention with respect to repeated indices is employed, that is, $a_{i} b_{i}=\sum_{i=1}^{3} a_{i} b_{i}$. Moreover, we denote by $a \cdot b=a_{i} b_{i}$ the inner product of the vectors $a=\left(a_{i}\right)$ and $b=\left(b_{i}\right)$. The upper subscript ${ }^{\top}$ represents the transpose of a matrix or a vector. Given a function $\theta(\mathrm{x})$ defined in $\Omega$ we denote by $\theta_{, i}$ or $\partial_{i} \theta$ its partial derivative with respect to $x_{i}$, that is, $\theta_{, i}=\partial_{i} \theta=\frac{\partial \theta}{\partial x_{i}}$, and by $\theta_{, i j}$ or $\partial_{i j} \theta$ its second partial derivative with respect to $x_{i}$ and $x_{j}$, that is, $\theta_{, i j}=\partial_{i j} \theta=\frac{\partial^{2} \theta}{\partial x_{i} \partial x_{j}}$. We denote by $v=\left(v_{1}, v_{2}, v_{3}\right)$ the outward unit normal vector to $\partial \Omega$, by the same letter $v=\left(v_{1}, v_{2}\right)$ the outward unit normal vector to $\partial \omega$, and finally by $\partial_{v} \vartheta=v_{\alpha} \partial_{\alpha} \vartheta$ the outer normal derivative along $\partial \omega$ of $\vartheta: \omega \rightarrow \mathbb{R}$.

Now, let $\Xi$ represent any open subset of $\mathbb{R}^{n}$, with $n=2,3$. We define $\mathscr{D}(\Xi)$ to be the linear space of functions 
infinitely differentiable and with compact support on $\Xi$, and denote by $\mathscr{D}^{\prime}(\Xi)$ the dual space of $\mathscr{D}(\Xi)$, often called the space of distributions on $\Xi$. For $m=1$ or $m=2$ and $p=2$, the Sobolev spaces $H^{m}(\Xi)$ (also denoted by $\left.W^{m, 2}(\Xi)\right)$ are defined by

$$
\begin{aligned}
H^{1}(\Xi)= & \left\{v \in L^{2}(\Xi): \quad \partial_{i} v \in L^{2}(\Xi), \quad \text { for } i=1, \ldots, n\right\}, \\
H^{2}(\Xi)= & \left\{v \in L^{2}(\Xi): \quad \partial_{i} v, \quad \partial_{i j} v \in L^{2}(\Xi),\right. \\
& \text { for } i, j=1, \ldots, n\},
\end{aligned}
$$

where $L^{2}(\Xi)=\left\{v: \Xi \rightarrow \mathbb{R}, \quad \int_{\Xi}|v|^{2} \mathrm{~d} \Xi<+\infty\right\}$ and the partial derivatives are interpreted as distributional derivatives.

We suppose that a piezoelectric anisotropic and nonhomogeneous material occupies the bounded thin plate $\bar{\Omega} \subset \mathbb{R}^{3}$. We denote by $C=\left(C_{i j k l}\right), P=\left(P_{i j k}\right)$ and $\varepsilon=\left(\varepsilon_{i j}\right)$, respectively, the elastic (fourth-order) tensor field, the piezoelectric (third-order) tensor field, and the dielectric (second-order) tensor field that characterize the material properties. The coefficients $C_{i j k l}, P_{i j k}, \varepsilon_{i j}$ are sufficiently smooth functions defined in $\bar{\omega} \times[-h, h]$ that satisfy the following symmetry properties: $P_{i j k}=P_{i k j}, \varepsilon_{i j}=\varepsilon_{j i}, C_{i j k l}=$ $C_{i j k l}=C_{k l i j}$.

Moreover, the plate is clamped along $\Gamma_{D}$, and subject to an applied electric potential $\varphi_{0}$ on $\Gamma_{e D}\left(\varphi_{0}^{+}\right.$and $\varphi_{0}^{-}$are the restrictions of $\varphi_{0}$ to $\Gamma_{+}$and $\Gamma_{-}$, respectively). In addition, $f=\left(f_{i}\right): \Omega \rightarrow \mathbb{R}^{3}$ represents the density of the applied body forces acting on the plate $\bar{\Omega}, g=\left(g_{i}\right): \Gamma_{N} \rightarrow \mathbb{R}^{3}$ the density of the applied surface forces on $\Gamma_{N}\left(g^{+}\right.$and $g^{-}$ are the restriction of $g$ to $\Gamma_{+}$and $\Gamma_{-}$, respectively). We assume that there is neither electric charge in $\Omega$ (this means that the material is dielectric) nor on $\Gamma_{e N}$.

In the framework of small deformations and linear piezoelectricity, the three-dimensional static equations for the piezoelectric plate are the following: Find a displacement vector field $u: \Omega \rightarrow \mathbb{R}^{3}$ and an electric potential $\varphi: \Omega \rightarrow$ $\mathbb{R}^{3}$ such that

$\sigma_{i j}=C_{i j k l} e_{k l}(u)-P_{k i j} E_{k}(\varphi)$ in $\Omega$,

$D_{k}=P_{k i j} e_{i j}(u)+\varepsilon_{k l} E_{l}(\varphi)$ in $\Omega$,

$\sigma_{i j, j}=-f_{i} \quad$ in $\Omega$,

$D_{i, i}=0 \quad$ in $\Omega$,

$u=0 \quad$ on $\Gamma_{D}, \quad \sigma_{i j} v_{j}=g_{i} \quad$ on $\Gamma_{N}$,

$D_{i} v_{i}=0 \quad$ on $\Gamma_{e N}, \quad \varphi=\varphi_{0} \quad$ on $\Gamma_{e D}$.

In (1)-(6), $\sigma=\left(\sigma_{i j}\right): \Omega \rightarrow \mathbb{R}^{9}$ is the stress tensor field, $D=\left(D_{k}\right): \Omega \rightarrow \mathbb{R}^{3}$ the electric displacement vector field and $e(u)$ the linear strain tensor defined by

$e(u)=\left(e_{i j}(u)\right), \quad e_{i j}(u)=\frac{1}{2}\left(\partial_{i} u_{j}+\partial_{j} u_{i}\right)$

and $E(\varphi)$ is the electric vector field defined by

$E(\varphi)=\left(E_{i}(\varphi)\right), \quad E_{i}(\varphi)=-\partial_{i} \varphi$.

Eqs. (1) and (2) are the constitutive equations evidencing the electromechanical coupling, (3) represents the equilibrium mechanical equation, (4) the Maxwell-Gauss equa- tion, (5) are the displacement and traction boundary conditions, and finally (6) represents the electric boundary conditions.

\subsection{The 2D asymptotic piezoelectric anisotropic plate model}

Now, we apply the asymptotic analysis procedure to the variational formulation of the $3 \mathrm{D}$ piezoelectric anisotropic plate model (1)-(6). As the plate thickness $2 h$ approaches 0 , this $3 \mathrm{D}$ model leads to a limit model that is a reduced 2D model, henceforth called the 2D asymptotic piezoelectric anisotropic plate model, or shortly, the 2D asymptotic plate model. The arguments used to mathematically justify this limit model can be found in [11], see also [9]. Its variational formulation is the following

$\left\{\begin{array}{l}\text { Find }(u, \varphi) \in V_{\mathrm{KL}} \times \Psi_{l}, \quad \text { such that: } \\ a((u, \varphi),(v, \psi))=l(v, \psi) \quad \forall(v, \psi) \in V_{\mathrm{KL}} \times \Psi_{l 0}, \\ \varphi=\varphi_{0}, \quad \text { on } \Gamma_{ \pm} .\end{array}\right.$

Here $V_{\mathrm{KL}}$ is the Kirchhoff-Love mechanical displacement space defined by

$$
\begin{aligned}
& V_{\mathrm{KL}}=\left\{v=\left(v_{1}, v_{2}, v_{3}\right) \in\left[H^{1}(\Omega)\right]^{3}: \quad \exists \eta=\left(\eta_{1}, \eta_{2}, \eta_{3}\right)\right. \\
& \in\left[H^{1}(\omega)\right]^{2} \times H^{2}(\omega), \quad v_{\alpha}(x)=\eta_{\alpha}\left(x_{1}, x_{2}\right)-x_{3} \partial_{\alpha} \eta_{3}\left(x_{1}, x_{2}\right), \\
& \left.v_{3}(x)=\eta_{3}\left(x_{1}, x_{2}\right), \quad \eta_{1_{\mid \gamma_{0}}}=\eta_{\left.\right|_{\left.\right|_{0}}}=\eta_{3_{\mid \gamma_{0}}}=0, \quad \partial_{v} \eta_{3_{\mid \gamma_{0}}}=0\right\}
\end{aligned}
$$

and $\Psi_{l}, \Psi_{l 0}$ are the spaces associated to the admissible electric potentials defined by

$$
\begin{aligned}
& \Psi_{l}=\left\{\psi \in L^{2}(\Omega): \quad \partial_{3} \psi \in L^{2}(\Omega)\right\}, \\
& \Psi_{l 0}=\left\{\psi \in L^{2}(\Omega): \quad \partial_{3} \psi \in L^{2}(\Omega), \quad \psi_{\mid \Gamma_{ \pm}}=0\right\} .
\end{aligned}
$$

Moreover

$$
\begin{aligned}
a((u, \varphi),(v, \psi))= & \int_{\Omega} A_{\alpha \beta \gamma \rho} e_{\alpha \beta}(u) e_{\gamma \rho}(v) \mathrm{d} \Omega+\int_{\Omega} p_{33} \partial_{3} \varphi \partial_{3} \psi \mathrm{d} \Omega \\
& -\int_{\Omega} p_{3 \alpha \beta}\left[e_{\alpha \beta}(u) \partial_{3} \psi-e_{\alpha \beta}(v) \partial_{3} \varphi\right] \mathrm{d} \Omega,
\end{aligned}
$$

and

$l(v, \psi)=\int_{\Omega} f \cdot v \mathrm{~d} \Omega+\int_{\Gamma_{N}} g \cdot v \mathrm{~d} \Gamma_{N}$.

The modified coefficients $A_{\alpha \beta \gamma \rho}, p_{3 \alpha \beta}$ and $p_{33}$ depend only on $C_{i j k l}, P_{i j k}$ and $\varepsilon_{i j}$ and are defined by

$$
\begin{aligned}
& A_{\alpha \beta \gamma \rho}=C_{\alpha \beta \gamma \rho}-\frac{C_{\alpha \beta 33} C_{33 \gamma \rho}}{C_{3333}}+C_{\alpha \beta 33} \frac{C_{v 333}}{C_{3333}} b_{\delta v} a_{\delta \gamma \rho}-C_{\alpha \beta v 3} b_{\delta v} a_{\delta \gamma \rho}, \\
& p_{3 \alpha \beta}=P_{3 \alpha \beta}-\frac{C_{\alpha \beta 33}}{C_{3333}} P_{333}+C_{\alpha \beta 33} \frac{C_{33 v 3}}{C_{3333}} b_{\delta v} c_{\delta}-C_{\alpha \beta v 3} b_{\delta v} c_{\delta}, \\
& p_{33}=\varepsilon_{33}+\frac{P_{333} P_{333}}{C_{3333}}-P_{333} \frac{C_{33 v 3}}{C_{3333}} b_{\delta v} c_{\delta}+P_{3 v 3} b_{\delta v} c_{\delta},
\end{aligned}
$$

where

$$
\begin{aligned}
& a_{\delta \gamma \rho}=C_{33 \gamma \rho} C_{\delta 333}-C_{\delta 3 \gamma \rho} C_{3333}, \quad c_{\delta}=C_{\delta 333} P_{333}-C_{3333} P_{3 \delta 3}, \\
& {\left[b_{\delta v}\right]=\left[C_{\delta 333} C_{33 v 3}-C_{\delta 3 v 3} C_{3333}\right]^{-1}}
\end{aligned}
$$


where the latter equality is to be understood as identity between two matrices. We observe that this limit problem (7) is an extension of Theorem 3.3 in [11] (established for the case $C_{\alpha \beta \gamma 3}=0=C_{\alpha 333}$ ) to the general case of anisotropy. In fact, (7) differs from the corresponding limit problem defined in Theorem 3.3 of [11] with respect to the formulas (8) for the modified coefficients $A_{\alpha \beta \gamma \rho}, p_{3 \alpha \beta}$ and $p_{33}$. One easily checks that in case of $C_{\alpha \beta \gamma 3}=0=C_{\alpha 333}$, the formulas in (8) are identical with those given in Theorem 3.3 of [11]: the so-called reduced elastic coefficients $A_{\alpha \beta \gamma \rho}$ turn to (cf. formula (41) in [11])

$A_{\alpha \beta \gamma \rho}=C_{\alpha \beta \gamma \rho}-\frac{C_{\alpha \beta 33} C_{33 \gamma \rho}}{C_{3333}}$,

the modified piezoelectric coefficients $p_{3 \alpha \beta}$ and corresponding vector $p_{3}$ are now equal to (cf. formula (42) in [11])

$p_{3 \alpha \beta}=P_{3 \alpha \beta}-\frac{C_{\alpha \beta 33}}{C_{3333}} P_{333}, \quad p_{3}=\left[\begin{array}{lll}p_{311} & p_{322} & p_{312}\end{array}\right]$

and the scalar field $p_{33}$ is now (cf. formula (43) in [11])

$$
\begin{aligned}
p_{33}= & \varepsilon_{33}+\frac{P_{333} P_{333}}{C_{3333}}+\frac{1}{\operatorname{det}\left[\begin{array}{ll}
C_{1313} & C_{1323} \\
C_{2313} & C_{2323}
\end{array}\right]} \\
& \times\left[\begin{array}{c}
P_{323} \\
-P_{313}
\end{array}\right]^{\top}\left[\begin{array}{ll}
C_{1313} & C_{1323} \\
C_{2313} & C_{2323}
\end{array}\right]\left[P_{323}-P_{313}\right] .
\end{aligned}
$$

The procedure to obtain the more general formulas (8) for $A_{\alpha \beta \gamma \rho}, p_{3 \alpha \beta}$ and $p_{33}$ for the general case of anisotropy is the same as indicated in [11] (cf. Section 5 in [11]). It suffices to use the equations (35) in [11] with non-zero $C_{\alpha \beta \gamma 3}$ and $C_{\alpha, 333}$ to derive the new formulas for $\kappa_{i j}$ (cf. (34) in [11]) and subsequently introduce these $\kappa_{i j}$ in the two equations of formula (40) of [11]. The latter step results in the new formulas (8) for $A_{\alpha \beta \gamma \rho}, p_{3 \alpha \beta}$ and $p_{33}$.

Remark 2.1. In Section 3.3 we consider a laminated plate, whose layers are made of monoclinic piezoelectric materials with elastic, piezoelectric and dielectric coefficients that are independent of $x_{3}$ in each layer. Thus, the material of each layer satisfies $C_{\alpha \beta \gamma 3}=0=C_{\alpha 333}$, and therefore, for each layer the corresponding coefficients $A_{\alpha \beta \gamma \rho}, p_{3 \alpha \beta}$ and $p_{33}$ are defined by (9)-(11). It is also proven in [11], Theorem 3.3 , that for the case of a plate with $C_{\alpha \beta \gamma 3}=0=C_{\alpha 333}$, problem (7) admits a unique solution $(u, \varphi)$. One easily verifies that this uniqueness result also holds true for the variational Eq. (7), where we consider a laminated plate, whose layers are made of monoclinic piezoelectric materials, as that used in Section 3.3.

A straightforward computation shows that (7) can be reformulated: in fact, it is equivalent to an easier model, in which the Kirchhoff-Love displacement $u$ is the unique solution of a two-dimensional piezoelectric plate model defined on the plate's middle plane. Provided $u$ has been found, the electric potential $\varphi$ is an explicit function of the prescribed electric potential on the lower and upper surface and the tangential and transverse components of this Kirchhoff-Love mechanical displacement $u$. This result is stated in the next Theorem 2.1.

Theorem 2.1 (Equivalent reformulation of Problem (7)). Let $(u, \varphi) \in V_{\mathrm{KL}} \times \Psi_{l}$ be the unique solution of problem (7), where $u_{\alpha}=\xi_{\alpha}-x_{3} \partial_{\alpha} \xi_{3}, u_{3}=\xi_{3}$, and $\xi=\left(\xi_{1}, \xi_{2}, \xi_{3}\right)$. Then, the electric potential $\varphi$ satisfies

$$
\begin{aligned}
\varphi\left(x_{1}, x_{2}, x_{3}\right)= & \varphi_{0}^{-}\left(x_{1}, x_{2}\right)+\int_{-h}^{x_{3}}\left[\left(\frac{p_{3 \alpha \beta}}{p_{33}}-\frac{a_{\alpha \beta}}{p_{33}} c\right) e_{\alpha \beta}(\xi)\right. \\
& \left.-\left(\frac{p_{3 \alpha \beta}}{p_{33}} y_{3}-\frac{b_{\alpha \beta}}{p_{33}} c\right) \partial_{\alpha \beta} \xi_{3}+\frac{\varphi_{0}^{+}-\varphi_{0}^{-}}{p_{33}} c\right] \mathrm{d} y_{3},
\end{aligned}
$$

where $\varphi_{0}^{+}$and $\varphi_{0}^{-}$are the restrictions of $\varphi_{0}$ to $\Gamma_{+}$and $\Gamma_{-}$, respectively, and

$$
\begin{aligned}
& a_{\alpha \beta}=\int_{-h}^{+h} \frac{p_{3 \alpha \beta}}{p_{33}} \mathrm{~d} x_{3}, \quad b_{\alpha \beta}=\int_{-h}^{+h} x_{3} \frac{p_{3 \alpha \beta}}{p_{33}} \mathrm{~d} x_{3}, \\
& c=\left(\int_{-h}^{+h} \frac{1}{p_{33}} \mathrm{~d} x_{3}\right)^{-1}
\end{aligned}
$$

are functions defined on the middle plane $w$ of the plate. Moreover, $u \in V_{\mathrm{KL}}$ is the solution of the variational equation Find $u \in V_{\mathrm{KL}}$ such that : $\bar{a}(u, v)=\bar{l}(v) \quad \forall v \in V_{\mathrm{KL}}$,

where for any $v=\left(\eta_{1}-x_{3} \partial_{1} \eta_{3}, \eta_{2}-x_{3} \partial_{2} \eta_{3}, \eta_{3}\right) \in V_{\mathrm{KL}}$

$\bar{l}(v)=\int_{\Omega} f \cdot v \mathrm{~d} \Omega+\int_{\Gamma_{N}} g \cdot v \mathrm{~d} \Gamma_{N}-\int_{\Omega}\left(\varphi_{0}^{+}-\varphi_{0}^{-}\right) \frac{p_{3 \alpha \beta}}{p_{33}} c e_{\alpha \beta}(v) \mathrm{d} \Omega$,

and

$\bar{a}(u, v)=\int_{\omega}\left[N_{\alpha \beta}(u) e_{\alpha \beta}(\eta)+M_{\alpha \beta}(u) \partial_{\alpha \beta} \eta_{3}\right] \mathrm{d} \omega$.

Here, $\left(N_{\alpha \beta}(u)\right)$ and $\left(M_{\alpha \beta}(u)\right)$ are the components of secondorder tensor fields associated to the Kirchhoff-Love displacement $u$ given by the following matrix formula

$$
\left[\begin{array}{l}
N_{\alpha \beta}(u) \\
M_{\alpha \beta}(u)
\end{array}\right]=O\left[\begin{array}{l}
e_{\gamma \rho}(\xi) \\
\partial_{\gamma \rho} \xi_{3}
\end{array}\right]
$$

where the $6 \times 6$ matrix $O$ is (in general) non-symmetric. Its components are functions of the middle plane $\omega$, namely

$O=\left[\begin{array}{ll}\int_{-h}^{+h} B_{\alpha \beta \gamma \rho} \mathrm{d} x_{3} & -\int_{-h}^{+h} D_{\alpha \beta \gamma \rho} \mathrm{d} x_{3} \\ -\int_{-h}^{+h} x_{3} B_{\alpha \beta \gamma \rho} \mathrm{d} x_{3} & \int_{-h}^{+h} x_{3} D_{\alpha \beta \gamma \rho} \mathrm{d} x_{3}\end{array}\right]_{6 \times 6}$

with

$$
\begin{aligned}
& B_{\alpha \beta \gamma \rho}=A_{\alpha \beta \gamma \rho}+\frac{p_{3 \alpha \beta} p_{3 \gamma \rho}}{p_{33}}-\frac{p_{3 \alpha \beta} a_{\gamma \rho}}{p_{33}} c \\
& D_{\alpha \beta \gamma \rho}=x_{3} A_{\alpha \beta \gamma \rho}+x_{3} \frac{p_{3 \alpha \beta} p_{3 \gamma \rho}}{p_{33}}-\frac{p_{3 \alpha \beta} b_{\gamma \rho}}{p_{33}} c .
\end{aligned}
$$

In particular, the bilinear form $\bar{a}(.,$.$) in (16) is non-symmetric$ (if $O$ is non-symmetric), and the tangential $\left(\xi_{1}, \xi_{2}\right)$ and transverse $\xi_{3}$ components of the unknown displacement $u$ are coupled in (14). 
Proof. Considering $v=0$ in (7) we obtain

$\int_{\Omega}\left[p_{33} \partial_{3} \varphi-p_{3 \alpha \beta} e_{\alpha \beta}(u)\right] \partial_{3} \psi \mathrm{d} \Omega=0$.

Since $\mathscr{D}(\Omega)$ is dense in $\Psi_{l 0}$ (see, e.g., [9]), we can take $\psi \in \mathscr{D}(\Omega)$, which gives

$-\int_{\Omega} \partial_{3}\left[p_{33} \partial_{3} \varphi-p_{3 \alpha \beta} e_{\alpha \beta}(u)\right] \psi \mathrm{d} \Omega=0$.

Hence, $\partial_{3}\left[p_{33} \partial_{3} \varphi-p_{3 \alpha \beta} e_{\alpha \beta}(u)\right]=0$ and thus, there exists $d^{1} \in \mathscr{D}^{\prime}(\omega)$ such that

$-p_{33} \partial_{3} \varphi+p_{3 \alpha \beta} e_{\alpha \beta}(u)=d^{1} \quad$ in $\mathscr{D}^{\prime}(\omega)$,

or equivalently, because $e_{\alpha \beta}(u)=e_{\alpha \beta}(\xi)-x_{3} \partial_{\alpha \beta} \xi_{3}$,

$\partial_{3} \varphi=\frac{p_{3 \alpha \beta}}{p_{33}}\left[e_{\alpha \beta}(\xi)-x_{3} \partial_{\alpha \beta} \xi_{3}\right]-\frac{1}{p_{33}} d^{1}$.

After integration over $x_{3}$ this yields

$$
\begin{aligned}
\varphi\left(x_{1}, x_{2}, x_{3}\right)= & \varphi\left(x_{1}, x_{2},-h\right)+\int_{-h}^{x_{3}} \frac{p_{3 \alpha \beta}}{p_{33}}\left[e_{\alpha \beta}(\xi)\right. \\
& \left.-y_{3} \partial_{\alpha \beta} \xi_{3}\right] \mathrm{d} y_{3}-\int_{-h}^{x_{3}} \frac{1}{p_{33}} d^{1} \mathrm{~d} y_{3} .
\end{aligned}
$$

Since $\varphi\left(x_{1}, x_{2},-h\right)=\varphi_{0}^{-}\left(x_{1}, x_{2}\right)$ and $\varphi\left(x_{1}, x_{2},+h\right)=\varphi_{0}^{+}\left(x_{1}\right.$, $x_{2}$ ) we choose $x_{3}=+h$ in the previous expression to determine $d^{1}$ :

$$
\begin{aligned}
\varphi\left(x_{1}, x_{2},+h\right)= & \varphi\left(x_{1}, x_{2},-h\right)+\left(\int_{-h}^{+h} \frac{p_{3 \alpha \beta}}{p_{33}} \mathrm{~d} x_{3}\right) e_{\alpha \beta}(\xi) \\
& -\left(\int_{-h}^{+h} x_{3} \frac{p_{3 \alpha \beta}}{p_{33}} \mathrm{~d} x_{3}\right) \partial_{\alpha \beta} \xi_{3} \\
& -\left(\int_{-h}^{+h} \frac{1}{p_{33}} \mathrm{~d} x_{3}\right) d^{1},
\end{aligned}
$$

and clearly this equation is exactly the same as

$\varphi_{0}^{+}=\varphi_{0}^{-}+a_{\alpha \beta} e_{\alpha \beta}(\xi)-b_{\alpha \beta} \partial_{\alpha \beta} \xi_{3}-c^{-1} d^{1}$,

or equivalently

$d^{1}=c\left[\varphi_{0}^{-}-\varphi_{0}^{+}+a_{\alpha \beta} e_{\alpha \beta}(\xi)-b_{\alpha \beta} \partial_{\alpha \beta} \xi_{3}\right]$.

Finally, inserting this $d^{1}$ in (20) we directly obtain formula (12) for the electric potential.

Choosing now $\psi=0$ in (7) we get

$$
\begin{gathered}
\int_{\Omega} A_{\alpha \beta \gamma \rho} e_{\alpha \beta}(u) e_{\gamma \rho}(v) \mathrm{d} \Omega+\int_{\Omega} p_{3 \alpha \beta} e_{\alpha \beta}(v) \partial_{3} \varphi \mathrm{d} \Omega \\
=\int_{\Omega} f \cdot v \mathrm{~d} \Omega+\int_{\Gamma_{N}} g \cdot v \mathrm{~d} \Gamma_{N} .
\end{gathered}
$$

For the derivative $\partial_{3} \varphi$ given in (19) with $d^{1}$ as defined in (21) we have

$$
\begin{aligned}
\partial_{3} \varphi= & \left(\frac{p_{3 \alpha \beta}}{p_{33}}-\frac{a_{\alpha \beta}}{p_{33}} c\right) e_{\alpha \beta}(\xi)-\left(\frac{p_{3 \alpha \beta}}{p_{33}} x_{3}-\frac{b_{\alpha \beta}}{p_{33}} c\right) \partial_{\alpha \beta} \xi_{3} \\
& +\frac{\varphi_{0}^{+}-\varphi_{0}^{-}}{p_{33}} c
\end{aligned}
$$

and introducing this latter formula in (22) we obtain

$$
\begin{aligned}
& \int_{\Omega}\left[B_{\alpha \beta \gamma \rho} e_{\alpha \beta}(\xi)-D_{\alpha \beta \gamma \rho} \partial_{\gamma \rho}\left(\xi_{3}\right)\right]\left(e_{\alpha \beta}(\eta)-x_{3} \partial_{\alpha \beta} \eta_{3}\right) \mathrm{d} \Omega \\
& =\int_{\Omega} f \cdot v \mathrm{~d} \Omega+\int_{\Gamma_{N}} g \cdot v \mathrm{~d} \Gamma_{N}-\int_{\Omega}\left(\varphi_{0}^{+}-\varphi_{0}^{-}\right) \frac{p_{3 \alpha \beta}}{p_{33}} c e_{\alpha \beta}(v) \mathrm{d} \Omega,
\end{aligned}
$$

which is precisely the variational Eq. (14).

For later use we remark that for the last term on the right-hand side of (23) holds

$$
\begin{aligned}
& \int_{\Omega}\left(\varphi_{0}^{+}-\varphi_{0}^{-}\right) \frac{p_{3 \alpha \beta}}{p_{33}} c e_{\alpha \beta}(v) \mathrm{d} \Omega \\
& \quad=\int_{\omega}\left(\varphi_{0}^{+}-\varphi_{0}^{-}\right) c\left(a_{\alpha \beta} e_{\alpha \beta}(\eta)-b_{\alpha \beta} \partial_{\alpha \beta} \eta_{3}\right) \mathrm{d} \omega .
\end{aligned}
$$

In Corollary 2.1 we show that Theorem 2.1 is a generalization of Theorem 3.4 in [11].

Corollary 2.1 (Theorem 3.4 of [11]). Suppose that $C_{\alpha \beta \gamma 3}=$ $0=C_{\alpha 333}$ and in each layer the coefficients $p_{3 \alpha \beta}$ and $p_{33}$ are independent of $x_{3}$. Then (12) becomes

$$
\begin{aligned}
\varphi\left(x_{1}, x_{2}, x_{3}\right)= & \varphi_{0}^{-}\left(x_{1}, x_{2}\right) \\
& +\int_{-h}^{x_{3}}\left[-\left(\frac{p_{3 \alpha \beta}}{p_{33}} y_{3}\right) \partial_{\alpha \beta} \xi_{3}+\frac{\varphi_{0}^{+}-\varphi_{0}^{-}}{2 h}\right] \mathrm{d} y_{3},
\end{aligned}
$$

which is precisely the formula (88) of [11] after integration with respect to the thickness variable. Moreover, problem (14) coincides with problem (56) of [11] (we remark that $h=1$ in (56) of [11]), because in this case

$$
\bar{l}(v)=\int_{\Omega} f \cdot v \mathrm{~d} x+\int_{\Gamma_{N}} g \cdot v \mathrm{~d} \Gamma_{N}-\int_{\Omega} \frac{\varphi_{0}^{+}-\varphi_{0}^{-}}{2 h} p_{3 \alpha \beta} e_{\alpha \beta}(v) \mathrm{d} x,
$$

and

$\bar{a}(u, v)=\int_{\omega}\left[N_{\alpha \beta}(u) e_{\alpha \beta}(\eta)+M_{\alpha \beta}(u) \partial_{\alpha \beta} \eta_{3}\right] \mathrm{d} \omega$,

where $\left(N_{\alpha \beta}(u)\right)$ and $\left(M_{\alpha \beta}(u)\right)$ are defined by the following matrix formula

$$
\begin{aligned}
& {\left[\begin{array}{l}
N_{\alpha \beta}(u) \\
M_{\alpha \beta}(u)
\end{array}\right]=O\left[\begin{array}{l}
e_{\gamma \rho}(\xi) \\
\partial_{\gamma \rho} \xi_{3}
\end{array}\right]} \\
& =\left[\begin{array}{ll}
\int_{-h}^{+h} A_{\alpha \beta \gamma \rho} \mathrm{d} x_{3} & -\int_{-h}^{+h} x_{3} A_{\alpha \beta \gamma \rho} \mathrm{d} x_{3} \\
-\int_{-h}^{+h} x_{3} A_{\alpha \beta \gamma \rho} \mathrm{d} x_{3} & \int_{-h}^{+h}\left(x_{3}\right)^{2}\left(A_{\alpha \beta \gamma \rho}+\frac{p_{3 \alpha \beta} p_{3 \gamma \rho}}{p_{33}}\right) \mathrm{d} x_{3}
\end{array}\right]\left[\begin{array}{l}
e_{\gamma \rho}(\xi) \\
\partial_{\gamma \rho} \xi_{3}
\end{array}\right]
\end{aligned}
$$

with $A_{\alpha \beta \gamma \rho}, p_{3 \alpha \beta}$ and $p_{33}$ given by (9)-(11).

Proof. In fact, if $p_{3 \alpha \beta}$ and $p_{33}$ are independent of $x_{3}$, then $a_{\alpha \beta}=2 h \frac{p_{3 \alpha \beta}}{p_{33}}, \quad b_{\alpha \beta}=0, \quad c=\frac{p_{33}}{2 h}$, 
and

$\frac{p_{3 \alpha \beta}}{p_{33}}-\frac{a_{\alpha \beta}}{p_{33}} c=\frac{p_{3 \alpha \beta}}{p_{33}}-2 h \frac{p_{3 \alpha \beta}}{p_{33}^{2}} \frac{p_{33}}{2 h}=0$,

therefore (12) turns to (25), and (26) is obtained from (15) replacing $c$ by $\frac{p_{33}}{2 h}$. We also have

$\frac{p_{3 \alpha \beta} p_{3 \gamma \rho}}{p_{33}}-\frac{p_{3 \alpha \beta} a_{\gamma \rho}}{p_{33}} c=\frac{p_{3 \alpha \beta} p_{3 \gamma \rho}}{p_{33}}-\frac{p_{3 \alpha \beta}}{p_{33}} \frac{2 h p_{3 \gamma \rho}}{p_{33}} \frac{p_{33}}{2 h}=0$.

Thus, the coefficients $B_{\alpha \beta \gamma \rho}$ and $D_{\alpha \beta \gamma \rho}$ defined in (18) are equal to

$B_{\alpha \beta \gamma \rho}=A_{\alpha \beta \gamma \rho}, \quad D_{\alpha \beta \gamma \rho}=x_{3}\left(A_{\alpha \beta \gamma \rho}+\frac{p_{3 \alpha \beta} p_{3 \gamma \rho}}{p_{33}}\right)$.

Consequently the bilinear formula (16)-(18) turns to (27) and (28) with the coefficients $B_{\alpha \beta \gamma \rho}$ and $B_{\alpha \beta \gamma \rho}$ defined by (29).

We emphasize that Theorem 2.1 is a generalization of the results found in $[9,11]$ to the case of a completely non-homogeneous anisotropic piezoelectric plate. In particular the explicit formula (12) for the electric potential now depends both on the tangential and transverse components of the mechanical displacement. This formula is not found in $[9,11]$ where it is assumed that the material is mechanically isotropic and monoclinic, respectively, and where it is also supposed that the modified piezoelectric coefficients are independent of the thickness variable. Moreover it is worth mentioning that a similar asymptotic procedure as the one used to obtain the model described in Theorem 2.1 (which relies on the method developed formerly in [5] for elastic plates) was applied in $[7,8]$ to derive asymptotic models for anisotropic piezoelectric thin plates. But the latter models differ from the one described in Theorem 2.1 of the present paper. Namely the asymptotic electric potentials are not the same. This is due to different scaling techniques, different types of boundary conditions and also different types of anisotropic materials. In [7] the first-order asymptotic electric potential satisfies a twodimensional Poisson-Neumann problem with an effective dielectric constant accounting for electromechanical couplings. In [8] it is found that the asymptotic electric potential has two different formulas depending on the type of the electric boundary conditions - a short-circuited or an insulated plate. For the case of a short-circuited plate (this means the plate is submitted to prescribed electric potentials on the upper and lower faces and to a surface electric charge on its lateral surface) the asymptotic electric potential is a quadratic polynomial of the thickness variable $x_{3}$ (and that coincides with formula (25) of the present paper when the material is mechanically monoclinic and the modified piezoelectric coefficients are independent of the thickness variable). For the case of an insulated plate (that is a plate which is electric charge-free on whole its boundary) the asymptotic electric potential is constant in the plate's thickness variable.

\subsection{The laminated piezoelectric plate model}

In this subsection, the 2D asymptotic plate model defined in Theorem 2.1 is applied to a thin laminated plate made of several stacked layers of different piezoelectric anisotropic materials. We assume that, in each layer the elastic, piezoelectric and dielectric coefficients are independent of $x_{3}$. This special material structure enables particular formulas for the functions, matrices and vectors involved in the definition of the $2 \mathrm{D}$ asymptotic plate model of Theorem 2.1. Below, we give the detailed form for the matrix $O$ and the electric potential $\varphi$.

\subsubsection{The matrix $O$}

As before, the global plate $\bar{\Omega}=\bar{\omega} \times[-h, h]$, has middle plane $w \subset \mathbb{R}^{2}$ and global thickness $2 h$. The material and geometric properties of each lamina are indexed by the letter $s$. We assume that there are $k$ laminas, numbered from the lower face to the upper face of the global plate $\bar{\Omega}$. We do not impose any geometrical symmetry in the distribution of these $k$ laminas with respect to the middle plane $w$ of the global plate. Let $t_{s}$ be the thickness of lamina $s$ and $\left|z_{s}\right|$ the distance from $w$ to the middle plane of lamina $s$, measured along the axis $\mathrm{OX}_{3}$, where $z_{s}$ is positive if lamina $s$ is above $w$ and negative if it is below. In particular, the sum of the thicknesses of the $k$ laminas must be equal to $2 h$, that is $\sum_{s=1}^{k} t_{s}=2 h$. In this setting, the coefficients $a_{\alpha \beta}, b_{\alpha \beta}$ and $c$ introduced in (13) become

$$
\begin{aligned}
& a_{\alpha \beta}=\int_{-h}^{+h} \frac{p_{3 \alpha \beta}}{p_{33}} \mathrm{~d} x_{3}=\sum_{s=1}^{k} \frac{p_{3 \alpha \beta}^{s}}{p_{33}^{s}} t_{s}, \\
& b_{\alpha \beta}=\int_{-h}^{+h} x_{3} \frac{p_{3 \alpha \beta}}{p_{33}} \mathrm{~d} x_{3}=\sum_{s=1}^{k} \frac{p_{3 \alpha \beta}^{s}}{p_{33}^{s}} z_{s} t_{s}, \\
& c=\left(\int_{-h}^{+h} \frac{1}{p_{33}} \mathrm{~d} x_{3}\right)^{-1}=\left(\sum_{s=1}^{k} \frac{t_{s}}{p_{33}^{s}}\right)^{-1},
\end{aligned}
$$

and for the components of the matrix $O$ in (17) we get

$$
\begin{aligned}
& \int_{-h}^{+h} B_{\alpha \beta \gamma \rho} \mathrm{d} x_{3}=\sum_{s=1}^{k} \underbrace{\left[A_{\alpha \beta \gamma \rho}^{s}+\frac{p_{3 \alpha \beta}^{s} p_{3 \gamma \rho}^{s}}{p_{33}^{s}}-\frac{p_{3 \alpha \beta}^{s} a_{\gamma \rho}}{p_{33}^{s}} c\right]}_{B_{\alpha \beta \gamma \rho}^{s}} t_{s}, \\
& \int_{-h}^{+h} x_{3} B_{\alpha \beta \gamma \rho} \mathrm{d} x_{3}=\sum_{s=1}^{k} \underbrace{\left[A_{\alpha \beta \gamma \rho}^{s}+\frac{p_{3 \alpha \beta \beta}^{s} p_{3 \gamma \rho}^{s}}{p_{33}^{s}}-\frac{p_{3 \alpha \beta}^{s} a_{\gamma \rho}}{p_{33}^{s}} c\right]}_{B_{\alpha \beta \gamma \rho}^{s}} t_{s} z_{s}, \\
& \int_{-h}^{+h} D_{\alpha \beta \gamma \rho} \mathrm{d} x_{3}=\sum_{s=1}^{k} \underbrace{\left[A_{\alpha \beta \gamma \rho}^{s}+\frac{p_{3 \alpha \beta}^{s} p_{3 \gamma \rho}^{s}}{p_{33}^{s}}\right]}_{D_{\alpha \beta \gamma \rho}^{1 s}} t_{s} z_{s}-\sum_{s=1}^{k} \underbrace{\left[\frac{p_{3 \alpha \beta}^{s} b_{\gamma \rho}}{p_{33}^{s}} c\right]}_{D_{\alpha \beta \gamma \rho}^{2 s}} t_{s},
\end{aligned}
$$

and 


$$
\begin{aligned}
\int_{-h}^{+h} x_{3} D_{\alpha \beta \gamma \rho} \mathrm{d} x_{3}= & \sum_{s=1}^{k} \underbrace{\left[A_{\alpha \beta \gamma \rho}^{s}+\frac{p_{3 \alpha \beta}^{s} p_{3 \gamma \rho}^{s}}{p_{33}^{s}}\right]}_{D_{\alpha \beta \gamma \rho}^{1 s}} \frac{1}{12}\left(t_{s}^{3}+12 t_{s} z_{s}^{2}\right) \\
& -\sum_{s=1}^{k} \underbrace{\left[\frac{p_{3 \alpha \beta}^{s} b_{\gamma \rho}}{p_{33}^{s}} c\right]}_{D_{\alpha \beta \gamma \rho}^{2 s}} t_{s} z_{s} .
\end{aligned}
$$

Therefore, the matrix $O$ as defined in (17) becomes a sum $O^{\text {lam }}$ of (in general non-symmetric) matrices, namely

$$
O^{\mathrm{lam}}=\sum_{s=1}^{k}\left[\begin{array}{cc}
B_{\alpha \beta \gamma \rho}^{s} t_{s} & -D_{\alpha \beta \gamma \rho}^{1 s} t_{s} z_{s}+D_{\alpha \beta \gamma \rho}^{2 s} t_{s} \\
-B_{\alpha \beta \gamma \rho}^{s} t_{s} z_{s} & D_{\alpha \beta \gamma \rho}^{1 s} \frac{1}{12}\left(t_{s}^{3}+12 t_{s} z_{s}^{2}\right)-D_{\alpha \beta \gamma \rho}^{2 s} t_{s} z_{s}
\end{array}\right]_{6 \times 6} .
$$

This matrix (31) induces the bilinear form $\bar{a}(.,$.$) in (16) for$ the laminated plate. Note that the third term on the righthand side of the linear form $\bar{l}(\cdot)$ in (15) is defined by (24), where the coefficients $a_{\alpha \beta}, b_{\alpha \beta}$ and $c$ are given by (30).

\subsubsection{The electric potential $\varphi$}

Let us now turn to the formula of the electric potential for the case of the laminated plate. If $x_{3}$ belongs to lamina $i$, with $1 \leqslant i \leqslant k$, we obtain from (12)

$$
\begin{aligned}
\varphi\left(x_{1}, x_{2}, x_{3}\right)= & \varphi_{0}^{-}\left(x_{1}, x_{2}\right)+\sum_{s=1}^{i-1}\left[\left(\frac{p_{3 \alpha \beta}^{s}}{p_{33}^{s}}-\frac{a_{\alpha \beta}}{p_{33}^{s}} c\right) t_{s} e_{\alpha \beta}(\xi)\right. \\
& \left.-\left(t_{s} z_{s} \frac{p_{3 \alpha \beta}^{s}}{p_{33}^{s}}-t_{s} \frac{b_{\alpha \beta}}{p_{33}^{s}} c\right) \partial_{\alpha \beta} \xi_{3}+\frac{\varphi_{0}^{+}-\varphi_{0}^{-}}{p_{33}^{s}} c t_{s}\right] \\
& +\left(\frac{p_{3 \alpha \beta}^{i}}{p_{33}^{i}}-\frac{a_{\alpha \beta}}{p_{33}^{i}} c\right)\left(x_{3}-z_{i}+\frac{t_{i}}{2}\right) e_{\alpha \beta}(\xi) \\
& -\frac{p_{3 \alpha \beta}^{i}}{p_{33}^{i}}\left(x_{3}^{2}-\left(z_{i}-\frac{t_{i}}{2}\right)^{2}\right) \frac{1}{2} \partial_{\alpha \beta} \xi_{3} \\
& +\frac{b_{\alpha \beta}}{p_{33}^{i}} c\left(x_{3}-z_{i}+\frac{t_{i}}{2}\right) \partial_{\alpha \beta} \xi_{3}+\frac{\varphi_{0}^{+}-\varphi_{0}^{-}}{p_{33}^{i}} c\left(x_{3}-z_{i}+\frac{t_{i}}{2}\right) .
\end{aligned}
$$

Of course, if $x_{3}$ belongs to lamina 1 , the sum $\sum_{s=1}^{i-1}[\cdots]$ on the right-hand side of (32) disappears. We also remark that $\varphi$ is a quadratic polynomial of the thickness variable.

\section{Numerical study of actuator and sensor effects}

In this second part, we discretize the model obtained in Section 2.3 using standard finite elements. Moreover, by means of mulitiobjective optimization problems we numerically analyze actuator and sensor capabilities of this laminated piezoelectric plate model.

\subsection{The discrete model}

Applying the finite element method to (14) and (12) leads to a discrete laminated piezoelectric plate model, which is stated in the below Theorem 3.1. In the sequel we assume that the plate's middle plane is a rectangular domain $\omega$ that is discretized using $m=n_{1} n_{2}$ axis-parallel rectangles $\omega^{e}$, i.e., $\omega=\bigcup_{e=1}^{m} \omega^{e}$. We suppose $\omega^{e}=$ $\left[a_{1}^{e}, b_{1}^{e}\right] \times\left[c_{2}^{e}, d_{2}^{e}\right]$ and denote $h_{1}^{e}=b_{1}^{e}-a_{1}^{e}$ and $h_{2}^{e}=d_{2}^{e}-c_{2}^{e}$, that is, $\left\{\omega^{e}\right\}$ is affine equivalent to the reference element $\hat{\omega}=[-1,+1] \times[-1,+1]$.

Bilinear and non-conforming higher-order finite elements (cf. [23]) are chosen to approximate the tangential and transverse displacement fields $\left(\xi_{1}, \xi_{2}\right)$ and $u_{3}=\xi_{3}$ of the Kirchhoff-Love displacement $u$, respectively. Eight degrees of freedom of the bilinear finite element are the values of $\left(\xi_{1}, \xi_{2}\right)$ at the vertices of $\omega^{e}$, and the 12 degrees of freedom characterizing the non-conforming finite element are the values of $u_{3}, u_{3,1}$ and $u_{3,2}$ at the vertices of $\omega^{e}$. We also utilize the $2 \times 8$-matrix $M$ and the $12 \times 1$-vector $N^{e}$ that correspond, respectively, to the four shape functions of the bilinear finite element and the twelve shape functions corresponding to the non-conforming finite element defined in $\hat{\omega}$ (cf. (26) and (27) in [4]). Moreover, let $L^{e}$ and $S^{e}$ be the matrices that correspond to the derivatives of the shape functions of the bilinear and the non-conforming finite elements, respectively (cf. (38) and (39) in [4]).

If $n$ is the number of nodes in the finite element mesh, as approximation of the displacements $\left(\xi_{1}, \xi_{2}, \xi_{3}, \xi_{3,1}, \xi_{3,2}\right)$ in $\omega$ we obtain the vector $u \in \mathbb{R}^{5 n}$ defined by

$u=\left[\begin{array}{ll}u_{t g} & u_{t v}\end{array}\right] \in \mathbb{R}^{2 n+3 n} \quad$ with
$u_{t g}=\left(u_{1 j}, u_{2 j}\right)_{j=1}^{n}, \quad u_{t v}=\left(u_{3 j}, u_{31 j}, u_{32 j}\right)_{j=1}^{n}$,

where $u_{t g}$ and $u_{t v}$ are, respectively, the approximations of the tangential and transverse displacements $\left(\xi_{1}, \xi_{2}\right)$ and $\left(\xi_{3}, \xi_{3,1}, \xi_{3,2}\right)$. This means that $u_{1 j}, u_{2 j}$ and $u_{3 j}, u_{31 j}, u_{32 j}$ are the approximations of $\xi_{1}, \xi_{2}$ and $\xi_{3}, \xi_{3,1}, \xi_{3,2}$, respectively, at the node $j$ of the finite element mesh $\omega$. Moreover, if $P$ is an arbitrary set of indices, we denote by $u_{t v P}, u_{t g_{P}}$ the subvectors of $u_{t v}$ and $u_{t g}$ respectively, whose components have their indices in $P$.

Let also

$$
\begin{aligned}
& F_{i}=\int_{-h}^{+h} f_{i} \mathrm{~d} x_{3}+g_{i}^{+}+g_{i}^{-}, \quad \text { for } i=1,2,3, \\
& f_{t g}=\left[\begin{array}{ll}
F_{1} & F_{2}
\end{array}\right]^{\top} \quad \text { and } f_{t v}=F_{3}
\end{aligned}
$$

be the vectors associated to the density of the mechanical forces acting on the middle plane $\omega$ of the plate, and let the vectors $p_{3}^{s}, a^{\text {lam }}$ and $b^{\text {lam }}$ (related to the material coefficients $p_{3}, a_{\alpha \beta}$ and $b_{\alpha \beta}$ of the laminated plate, cf. (10) and (30)) be defined by

$p_{3}^{s}=\left[\begin{array}{lll}p_{311}^{s} & p_{322}^{s} & p_{312}^{s}\end{array}\right]$ for each layer $s$,

$$
a^{\mathrm{lam}}=\left[\begin{array}{lll}
a_{11} & a_{22} & a_{12}
\end{array}\right], \quad b^{\mathrm{lam}}=\left[\begin{array}{lll}
b_{11} & b_{22} & b_{12}
\end{array}\right] .
$$

Then we have the following theorem.

Theorem 3.1. The finite element discrete problem associated to (14) is

$\left\{\begin{array}{l}\text { Find } \quad u=\left[\begin{array}{ll}u_{t g} & u_{t v}\end{array}\right] \in \mathbb{R}^{5 n} \text { such that: } \\ u_{t g_{I}}=0, \quad u_{t v J}=0 \\ K u=F\end{array}\right.$ 
The equations $u_{t_{I}}=0$ and $u_{t v J}=0$ represent the discrete boundary conditions for the displacements. At the element level, the square matrix $K$ and the vector $F$ are defined by $K^{e}$ and $F^{e}$, respectively. The $20 \times 20$ matrix $K^{e}$ is in general non-symmetric and depends on the laminated material coefficients

$K^{e}=\frac{h_{1}^{e} h_{2}^{e}}{4} \int_{\hat{\omega}}\left(\left[\begin{array}{cc}L^{e^{\top}} & 0 \\ 0 & S^{e \top}\end{array}\right]_{20 \times 6} O_{6 \times 6}^{\operatorname{lam}}\left[\begin{array}{cc}L^{e} & 0 \\ 0 & S^{e}\end{array}\right]_{6 \times 20}\right) \mathrm{d} \omega^{e}$,

where $O^{\mathrm{lam}}$ is the material matrix defined in (31). The vector $F^{e}$ has 20 components and is related to the mechanical forces and the applied electric potential $\varphi_{0}^{+}$and $\varphi_{0}^{-}$. Assuming that the surface mechanical force $g=0$ in $\Gamma_{1}$ and $f_{\alpha}, g_{\alpha}^{+}, g_{\alpha}^{-}$are independent of $x_{3} \in[-h, h]$ we obtain

$$
\begin{aligned}
F^{e} & =\left[\begin{array}{c}
F_{t g}^{e} \\
F_{t v}^{e}
\end{array}\right], \text { where } \\
F_{t g}^{e} & =\frac{h_{1}^{e} h_{2}^{e}}{4} \int_{\hat{\omega}}\left[M^{\top} f_{t g}-\left(\varphi_{0}^{+}-\varphi_{0}^{-}\right) c L^{e \top} \sum_{s=1}^{k} \frac{p_{3}^{s \top}}{p_{33}^{s}} t_{s}\right] \mathrm{d} \hat{\omega}, \\
F_{t v}^{e} & =\frac{h_{1}^{e} h_{2}^{e}}{4} \int_{\hat{\omega}}\left[N^{e \top} f_{t v}+\left(\varphi_{0}^{+}-\varphi_{0}^{-}\right) c S^{e^{\top}} \sum_{s=1}^{k} \frac{p_{3}^{s \top}}{p_{33}^{s}} t_{s} z_{s}\right] \mathrm{d} \hat{\omega} .
\end{aligned}
$$

It is worth noticing that the nodal displacements $u_{t g}$ and $u_{t v}$ in (33) are coupled (due to the definition of $O^{\text {lam }}$ in (31)).

Furthermore, if $x_{3}$ belongs to lamina $i, 1 \leqslant i \leqslant k$, the finite element approximation of the electric potential (32) in $\omega^{e} \times(-h,+h)$ is defined by

$$
\begin{aligned}
\varphi\left(x_{1}, x_{2}, x_{3}\right)_{\mid \omega^{e} \times(-h,+h)} & \simeq \varphi_{0}^{-}+\sum_{s=1}^{i-1}\left[\left(\frac{p_{3}^{s}}{p_{33}^{s}}-\frac{a^{\mathrm{lam}}}{p_{33}^{s}} c\right) t_{s} L^{e} u_{t g}^{e}\right. \\
& \left.-\left(t_{s} z_{s} \frac{p_{3}^{s}}{p_{33}^{s}}-t_{s} \frac{b^{\mathrm{lam}}}{p_{33}^{s}} c\right) S^{e} u_{t v}^{e}+\frac{\varphi_{0}^{+}-\varphi_{0}^{-}}{p_{33}^{s}} c t_{s}\right] \\
& +\left(\frac{p_{3}^{i}}{p_{33}^{i}}-\frac{a^{\mathrm{lam}}}{p_{33}^{i}} c\right)\left(x_{3}-z_{i}+\frac{t_{i}}{2}\right) L^{e} u_{t g}^{e} \\
& -\frac{p_{3}^{i}}{p_{33}^{i}}\left(x_{3}^{2}-\left(z_{i}-\frac{t_{i}}{2}\right)^{2}\right) \frac{1}{2} S^{e} u_{t v}^{e}+\frac{b^{\mathrm{lam}}}{p_{33}^{i}} c\left(x_{3}-z_{i}+\frac{t_{i}}{2}\right) S^{e} u_{t v}^{e} \\
& +\frac{\varphi_{0}^{+}-\varphi_{0}^{-}}{p_{33}^{i}} c\left(x_{3}-z_{i}+\frac{t_{i}}{2}\right)
\end{aligned}
$$

and if $x_{3}$ belongs to lamina 1 , the sum $\sum_{s=1}^{i-1}[\ldots]$ disappears on the right-hand side of (35).

Proof. The arguments are similar to those used in Theorem 3.1 in [4], so we omit the proof. We remark that to obtain the above form, we have assumed that the surface mechanical force $g=0$ in $\Gamma_{1}$ and $f_{\alpha}, g_{\alpha}^{+}, g_{\alpha}^{-}$are independent of $x_{3} \in[-h, h]$ in order to simplify the formulas for the vector $F$. Otherwise the expression for $F^{e}$ in (34) would consist of more terms. Furthermore, to obtain (35), it suffices to use (32) and apply the following standard finite element approximations for each finite element $\omega^{e}$

$$
\begin{aligned}
& \left(\xi_{1}, \xi_{2}\right) \simeq M u_{t g}^{e}, \quad\left[e_{11}(\xi) e_{22}(\xi) 2 e_{12}(\xi)\right] \simeq L^{e} u_{t g}^{e} \\
& u_{3}=\xi_{3} \simeq N^{e} u_{t v}^{e}, \quad\left[\partial_{11} \xi_{3} \partial_{22} \xi_{3} 2 \partial_{12} \xi_{3}\right] \simeq S^{e} u_{t v}^{e}
\end{aligned}
$$

Remark 3.1. The finite element code for the discrete model described in the previous theorem is available on request (cf. http://www.mat.uc.pt/ isabelf/poci59502.html, code Lampiezo.m).

\subsection{Optimization problems}

We now describe the optimization problems that model the actuator and the sensor effect of the discrete 2D laminated piezoelectric plate model (defined in Theorem 3.1). For the actuator problem we vary the location of the applied electric potential difference $\varphi_{0}^{+}-\varphi_{0}^{-}$, and for the sensor problem the location of the applied mechanical loads. Moreover, for both problems, we may also change the order of the different materials and the thickness of each lamina. Before presenting the actuator and sensor optimization problems, we define the optimization variables.

\subsubsection{Optimization variables}

We consider three optimization variables: the vector $t$ of thicknesses, the vector mat of materials and the vector $l o c$ representing the location of the non-zero applied electric potential difference $\varphi_{0}^{+}-\varphi_{0}^{-}$or the non-zero applied mechanical loads $f_{t g}$ and $f_{t v}$. The vectors $t$ and mat are defined by

$$
\begin{aligned}
& t=\left(t_{1}, t_{2}, \ldots, t_{k}\right), \text { with } \sum_{s=1}^{k} t_{s}=2 h, t_{s}>0, \\
& m a t=\left(m_{1} t_{1}, \text { at }_{2}, \ldots, \text { mat }_{k}\right), \quad \text { mat }_{r} \neq \text { mat }_{s}, \quad \text { for } r=s+1 .
\end{aligned}
$$

The components of both vectors are numbered from the lower to the upper face of the laminated plate, and layers with zero thickness, or repeated materials are not allowed.

Next we define the vector loc. We assume that the nonzero applied electric potential differences or mechanical loads may act in regions of $\omega$ with the same size. These regions are numbered and the finite element discretization of $\omega$ is chosen such that the borders of the regions consist of edges of adjacent finite elements. Then, the optimization variable $l o c$ is defined by

$l o c=(i, j, p e)$,

where $1 \leqslant i \leqslant m_{j}$ is the number of regions of $\omega$ that consist of $j \geqslant 1$ adjacent finite elements $\left(m_{j}\right.$ is the total number of regions), where the non-zero electric potential difference or mechanical loads are applied. The set pe contains $i$ elements of $Y_{j}=\left\{1,2, \ldots, m_{j}\right\}$ representing the location of these regions. In particular, pe ranges over all subsets of $Y_{j}$ with cardinality $i$, that is $p e \in C_{i}^{m_{j}}\left(Y_{j}\right)$.

For example, for a rectangular mesh with $20 \times 20$ finite elements setting $l o c=(3,4 \times 4,[1,4,8])$ means that the non-zero applied electric potential difference or non-zero 
applied mechanical loads are acting in three regions of $\omega$, each consisting of $4 \times 4=16$ finite elements, located at the positions $p e=[1,4,8]$ of $Y_{16}=\left\{1, \ldots, m_{16}=25\right\}$.

Since the size of the regions with non-zero electric potential difference or mechanical load is independent of the finite element mesh, for finer meshes the number of adjacent finite elements $j$ corresponding to the regions in loc increases. Obviously, for a mesh with $m$ finite elements, $1 \leqslant m_{j} \leqslant m$ holds for any $j$ and $m_{j}=m$ for $j=1$.

\subsubsection{Actuator optimization problem}

The actuator effect of a piezoelectric material (also called the inverse piezoelectric effect) is the mechanical deformation generated by applying an external electric field to the material. The aim of this subsection is to present the optimization problem that focuses on the maximization of the actuator effect of the laminated piezoelectric plate model.

For a mesh with $m$ finite elements and $n$ global nodes, the mechanical displacement of the plate is determined by the displacements $\left(\xi_{1}, \xi_{2}, \xi_{3}\right)$ that define the KirchhoffLove displacement $u$ of the nodes in the plate's middle plane. For an arbitrary node $j$ in the middle plane's mesh, the corresponding three-dimensional displacement $\left(\xi_{1}, \xi_{2}\right.$, $\left.\xi_{3}\right)$ is approximated by $\left(u_{1 j}, u_{2 j}, u_{3 j}\right)$. Fixing the applied mechanical forces and the boundary conditions, the nodes' displacements depend on the location of the non-zero applied electric potential difference $l o c=(i, j, p e)$ as well as on the thickness and material vector $t=\left(t_{1}, \ldots, t_{k}\right)$ and $m a t=\left(m_{1} t_{1}, \ldots, m a t_{k}\right)$. Of course, for each fixed triple (loc, $t$, mat $)$ exists a node in the mesh that attains a maximum displacement $d(l o c, t, m a t)$, that is

$d($ loc,$t$, mat $)=\max _{j=1, \ldots, n}\left\|\left(u_{1 j}, u_{2 j}, u_{3 j}\right)\right\|_{\mathbb{R}^{3}}$,

where $\|\cdot\|_{\mathbb{R}^{3}}$ is the usual Euclidean norm in $\mathbb{R}^{3}$.

Our objective is to maximize $d($ loc,t, mat $)$ choosing appropriate $l o c=(i, j, p e), \quad t=\left(t_{1}, \ldots, t_{k}\right) \quad$ and $m a t=$ $\left(\right.$ mat $\left._{1}, \ldots, m t_{k}\right)$, where pe ranges over all the subsets of $Y_{j}$ with $i$ distinct elements. At the same time we want to minimize the number $i$ of regions of $\omega$ with non-zero electric potential difference. Therefore, two objectives are considered: the maximization of the displacements and the minimization of the number $i$ of regions. This corresponds to the following non-differentiable multi-objective actuator optimization problem

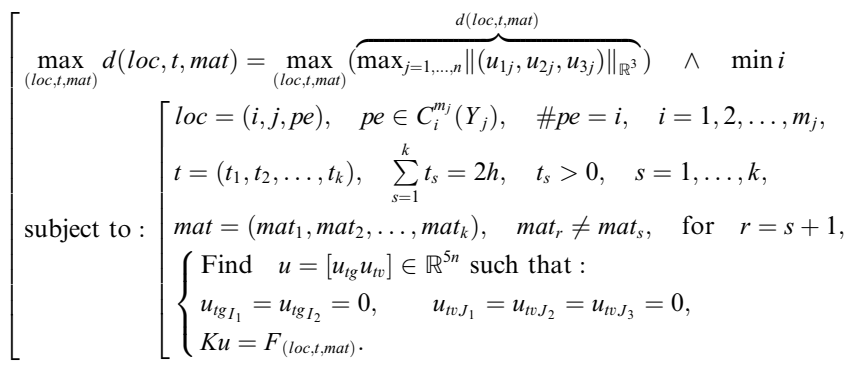

Note that the vector $F$ depends on (loc, $t, m a t)$, cf. (34). To emphasize this dependence, we write $F_{(l o c, t, m a t)}$ instead of $F$.

Note that for multi-objective problems such as (37), the aim is to characterize the set of so-called Pareto optimal solutions; these are solutions that cannot improve the performance of the first objective function (the node's displacement $d(l o c, t, m a t))$ without worsening the performance of the second one (the number $i$ of regions where the applied electric potential difference is non-zero) and vice-versa. If we drop the second objective, that is $\min i$, the multi-objective problem becomes an optimization problem with only one objective, namely to achieve a maximal node's displacement choosing (loc,t, mat) appropriately for fixed $i$ in $l o c$.

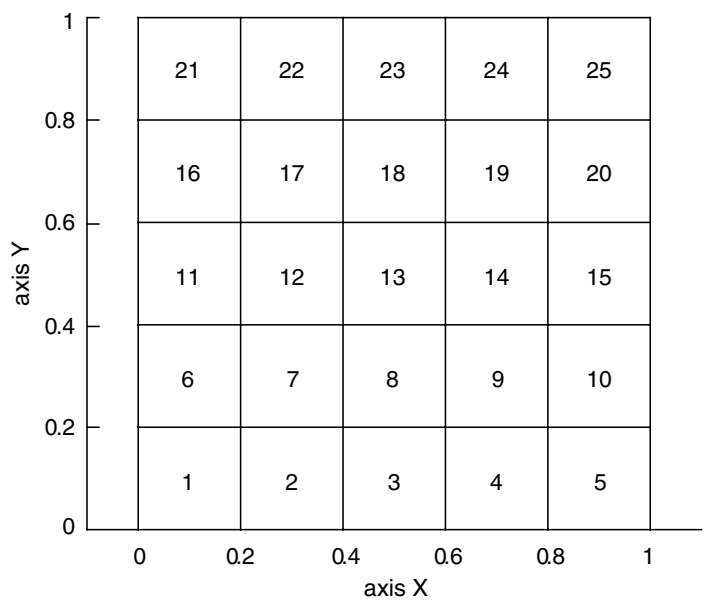

Fig. 1. Location $p e=[l]$ of each element $l \in Y_{16}=\{1, \ldots, 25\}$.

Table 1

Geometric, electric potential and mechanical loadings data

\begin{tabular}{llll}
\hline Parameter & Unit & Value (actuator problem) & Value (sensor problem) \\
\hline$L_{1}$ & $\mathrm{~m}$ & 1 & 1 \\
$L_{2}$ & $\mathrm{~m}$ & 1 & 1 \\
$h$ & $\mathrm{~m}$ & 0.01 & 0.01 \\
$\varphi_{0}^{+}$ & $\mathrm{V}$ & -100 & 0 \\
$\varphi_{0}^{-}$ & $\mathrm{V}$ & 0 & 0 \\
$f=\left(f_{i}\right)$ & $\mathrm{N}$ & $(0,0,0)$ & $(10,10,10)$ \\
$g=\left(g_{i}\right)$ & $\mathrm{N}$ & $(0,0,0)$ & $(10,10,10)$ \\
\hline
\end{tabular}

Table 2

Elastic, piezoelectric and dielectric data of the two materials

\begin{tabular}{llll}
\hline Parameter & Unit & PZT-5A ceramic value & PZT-5 ceramic value \\
\hline$E_{1}$ & $\mathrm{GPa}$ & 67 & 62 \\
$E_{2}=E_{3}$ & $\mathrm{GPa}$ & 67 & 54.9 \\
$v_{12}=v_{13}=v_{23}$ & & 0.31 & 0.31 \\
$G_{12}=G_{13}$ & $\mathrm{GPa}$ & 25.57 & 23.6 \\
$G_{23}$ & $\mathrm{GPa}$ & 25.57 & 18 \\
$P_{31}=P_{32}$ & $\mathrm{C} \mathrm{m}^{-2}$ & -9.30032142 & -12.006 \\
$P_{33}$ & $\mathrm{C} \mathrm{m}^{-2}$ & 20.3638 & 17.277 \\
$P_{15}=P_{26}$ & $\mathrm{C} \mathrm{m}^{-2}$ & 14.5749 & 15.812 \\
$\varepsilon_{33}$ & $\mathrm{~F} \mathrm{~m}^{-1}$ & $15.31742 \times 10^{-9}$ & $22.99 \times 10^{-9}$ \\
\hline
\end{tabular}


We point out that (37) is a combinatorial problem, since different combinations of the positions for the applied electric potentials, of the layer's thicknesses and the order of the materials lead to a different displacement of the nodes. In particular, the set $C_{i}^{m_{j}}\left(Y_{j}\right)$, that is, the admissible set for the optimization variable $p e$ is of cardinality $C_{i}^{m_{j}}=\frac{m_{j} !}{i !\left(m_{j}-i\right) !}$ (for instance, for a mesh with $m_{j}=25$ and $i=3$ we have $C_{3}^{25}=2300$ ). Even if the numbers $C_{i}^{m_{j}}$ can be reduced due to symmetries in the problem formulation, they become very large.

Obviously, the solutions of the optimization problem (37) strongly depend on the mechanical loadings and the boundary conditions imposed to the plate. In order to achieve a better understanding of the actuator effect, we assume that all the mechanical loadings $f=\left(f_{i}\right)$ and $g=\left(g_{i}\right)$ vanish. To analyze the influence of the boundary conditions, we consider a plate that is clamped on different parts of the lateral surface (this means that we vary the definition of the set $\left.\gamma_{0} \subset \partial \omega\right)$.

\subsubsection{Sensor optimization problem}

The sensor effect of a piezoelectric material (also called the direct piezoelectric effect) consists in the generation of an electric field in the material that is subject to an imposed mechanical force. In this subsection we describe the optimization problem related to the maximization of the sensor effect of the discrete laminated piezoelectric plate model. The optimization variables are those defined above, that is, $(l o c, t, m a t)$. As objective functional we choose the maximum value of the electric potential $\varphi$ (cf. (35)) at a predefined thickness $z_{s}$ for each lamina $s$. That is, for a mesh with $m$ finite elements we consider the non-differentiable function

$\operatorname{elpot}($ loc,$t$, mat $)=\max _{e=1, \ldots, m} \max _{s=1, \ldots, k}\left|\varphi_{\mid \omega^{e} \times\left\{z_{s}\right\}}\right|$.

We notice that the discrete electric potential $\varphi_{\mid \omega^{e} \times(-h,+h)}$ depends on (loc,t, mat) by means of the Kirchhoff-Love displacement $u$, which is the solution of $K u=F_{(l o c, t, m a t)}$, cf. (33) and (35).

Analogously to the actuator optimization problem, the objective is not only to maximize elpot (loc, $t$, mat), but also to minimize the number $i$ of regions of $\omega$ with non-zero mechanical forces. Therefore, two objectives are considered, which leads to the following sensor multi-objective optimization problem
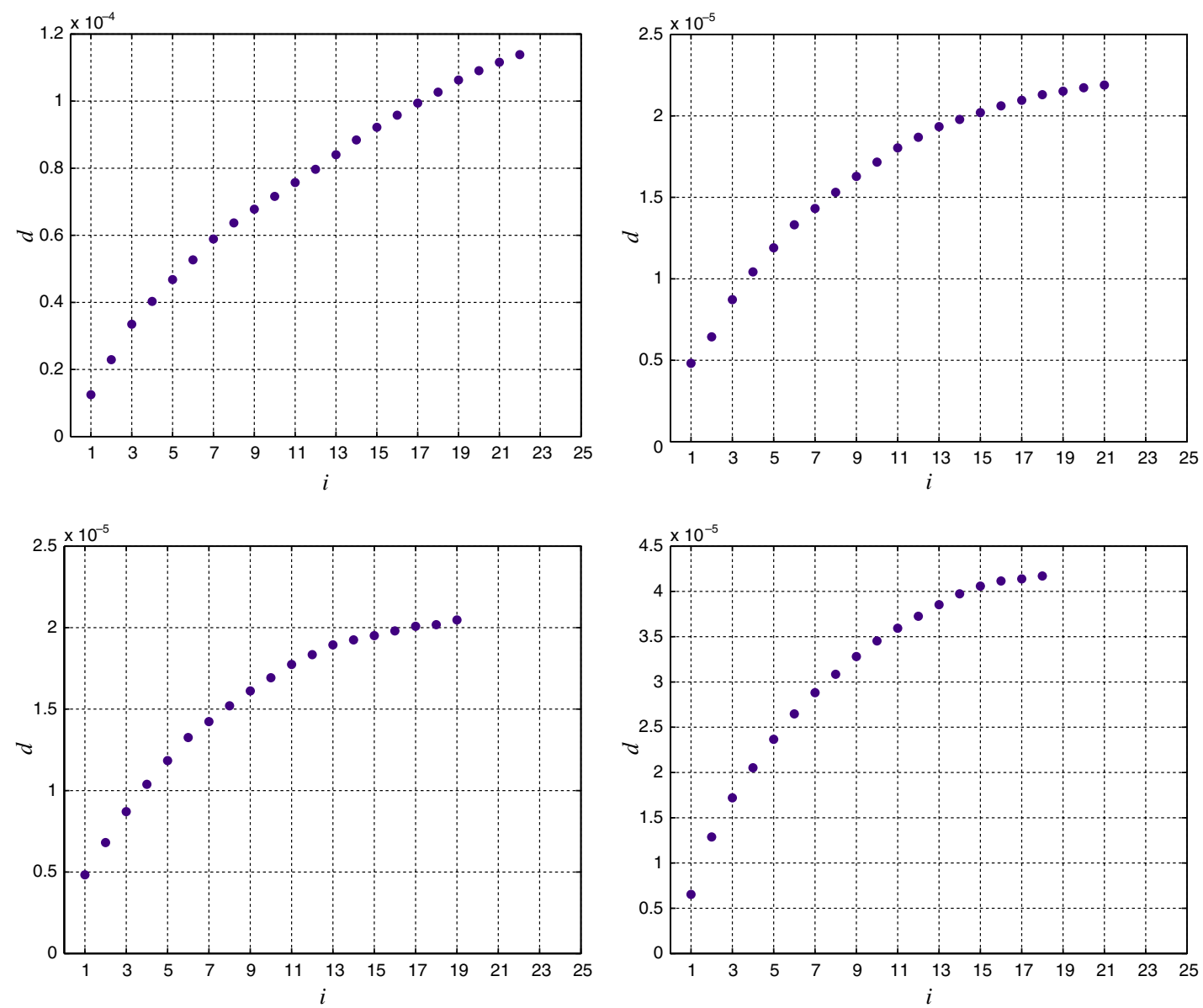

Fig. 2. Pareto curves: maximal displacement $d$ versus number of regions $i$ (where the electric potential difference $\varphi_{0}^{+}-\varphi_{0}^{-}$is applied) for the actuator multi-objective problem for $B C=1$ (upper left plot), $B C=2$ (upper right plot), $B C=3$ (lower left plot) and $B C=4$ (lower right plot). 


$$
\begin{aligned}
& {\left[\max _{(l o c, t, m a t)} \operatorname{elpot}(\text { loc, }, \text {, mat })=\max _{(l o c, t, m a t)}(\overbrace{\left.\max _{e=1, \ldots, m} \max _{s=1, \ldots, k} \mid \varphi_{\mid \omega^{e} \times\left\{z_{s}\right\}}\right\}}^{\text {elpot(loc,t,mat)}}) \wedge \min i\right.} \\
& \text { subject to : }\left[\begin{array}{l}
l o c=(i, j, p e), \quad p e \in C_{i}^{m_{j}}\left(Y_{j}\right), \quad \# p e=i, \quad i=1,2, \ldots, m_{j}, \\
t=\left(t_{1}, t_{2}, \ldots, t_{k}\right), \sum_{s=1}^{k} t_{s}=2 h, \quad t_{s}>0, \quad s=1, \ldots, k, \\
m^{m}=\left(\text { mat }_{1}, \text { mat }_{2}, \ldots, \text { mat }_{k}\right), \quad \text { mat }_{r} \neq \text { mat }_{s}, \quad \text { if } r=s+1, \\
\varphi_{\mid \omega \omega^{e} \times\left\{z_{s}\right\}} \text { defined in }(35) .
\end{array}\right.
\end{aligned}
$$

Unlike the actuator optimization problem we assume in this case non-zero mechanical forces and applied electric potential all nil.

\subsection{Numerical tests}

In this subsection, we describe the data and the solutions of our numerical tests. Moreover, we give a brief explanation of the genetic algorithms used to solve the multi-objective optimization problems (37) and (38).

\subsubsection{Data}

Let us consider a fixed three-dimensional coordinate system $O X Y Z$ and a laminated plate $\bar{\Omega}=\left[0, L_{1}\right] \times\left[0, L_{2}\right] \times$ $[-h,+h]$ with thickness $2 h$ and a rectangular middle plane $\omega=\left(0, L_{1}\right) \times\left(0, L_{2}\right)$. The set $\omega$ is partitioned into a mesh of $m$ sub-rectangles, where electrodes or mechanical loads are imposed. We assume a laminated plate consisting of two layers made of two different piezoelectric materials. The parameters $z_{s}$ and $t_{s}$ for $s=1,2$ (related to the thickness $t_{s}$ and introduced before in Section 2.3) are defined as

$z_{1}=-\frac{h+h_{0}}{2}+h_{0}, \quad t_{1}=h+h_{0}$,

$z_{2}=\frac{h-h_{0}}{2}+h_{0}, \quad t_{2}=h-h_{0}$,

where $h_{0} \in \mathbb{R}$ is such that $-h<h_{0}<h$. Layer 1 is below $\omega$ while layer 2 is above, and if $h_{0}=0$ then $t_{1}=t_{2}$ and both layers have the same thickness. If $h_{0}>0$ (respectively, $h_{0}<0$ ) layer 1 (respectively, layer 2) is thicker than layer 2 (respectively, layer 1 ).

In the sequel, we fix a $20 \times 20$ finite element mesh for the middle plane $\omega$; the finite elements and the nodes are numbered from the left side $l s=\{0\} \times\left[0, L_{2}\right]$ to the right side $r s=\left\{L_{1}\right\} \times\left[0, L_{2}\right]$ and from the bottom side $b s=$ $\left[0, L_{1}\right] \times\{0\}$ to the top side $t s=\left[0, L_{1}\right] \times\left\{L_{2}\right\}$ of $\omega$. We consider four types of clamped boundary conditions (abbreviation $B C$ ). If $B C=1, \omega$ is clamped only on the bottom side $\left(\gamma_{0}=b s\right)$; if $B C=2, \omega$ is clamped on the left, bottom and right sides $\left(\gamma_{0}=l s \cup b s \cup r s\right)$; if $B C=3, \omega$ is clamped on the two opposite left and right sides $\left(\gamma_{0}=l s \cup r s\right)$; finally, if $B C=4, \omega$ is clamped on the two consecutive bottom and right sides $\left(\gamma_{0}=b s \cup r s\right)$. We sup-
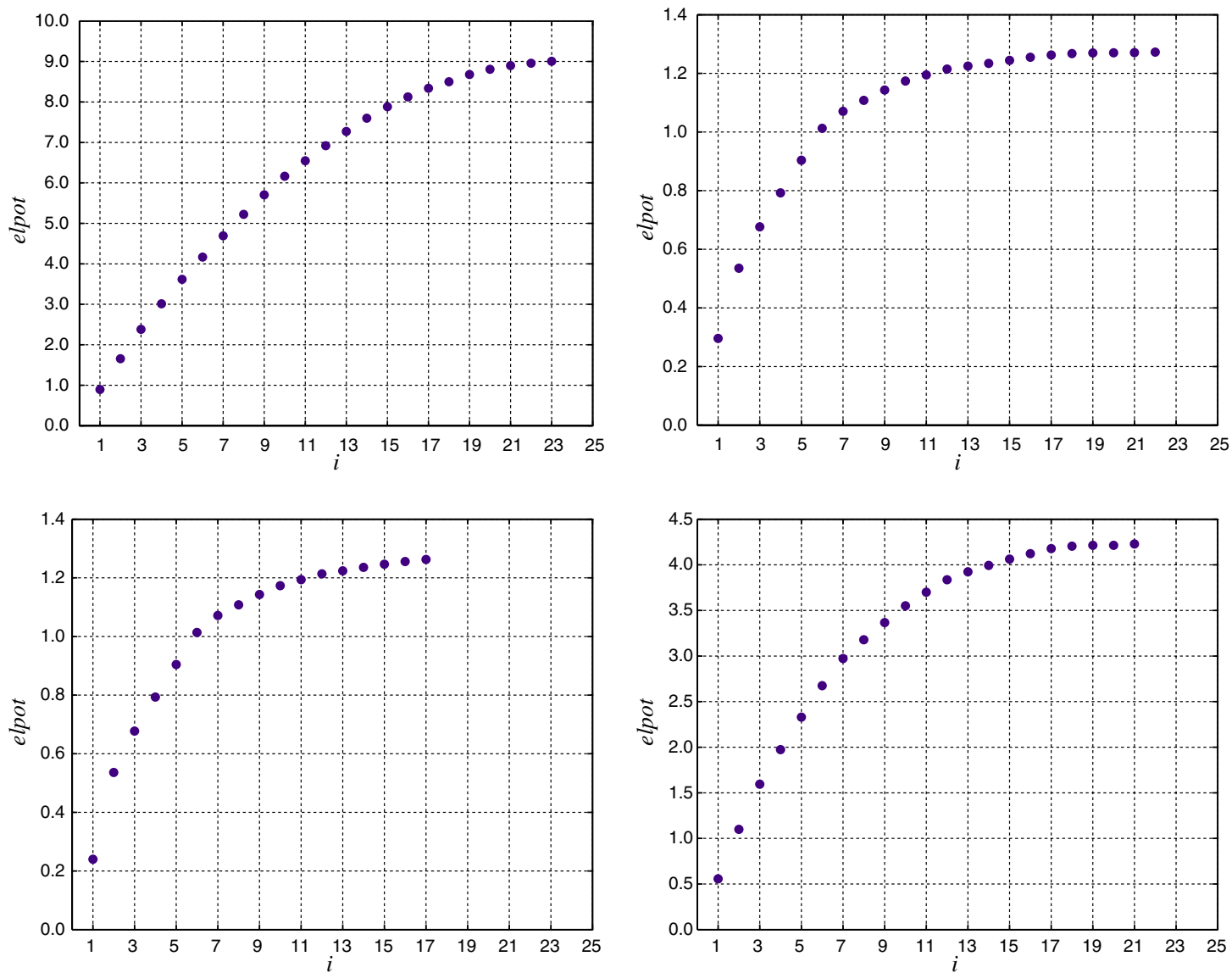

Fig. 3. Pareto curves: maximal electric potential elpot versus number of regions $i$ (where the mechanical loads are applied) for the sensor multi-objective problem for $B C=1$ (upper left plot), $B C=2$ (upper right plot), $B C=3$ (lower left plot) and $B C=4$ (lower right plot). 
pose that the non-zero applied electric potential difference (for actuator multi-objective problem (37)) or the non-zero applied mechanical loads (for the sensor multi-objective problem (38)) may act in $i=1$ up to $i=25$ regions consisting of $16=4 \times 4$ adjacent finite elements of the $20 \times 20$ mesh (we recall that the definition of $i$ in given in (36)), located at the positions pe $\subseteq Y_{16}=$ $\left\{1, \ldots, m_{16}=25\right\}$ as explained in Fig. 1 .

The exact data for the geometry, the electric potential and the mechanical loadings are given in Table 1.
The piezoelectric, dielectric and elastic coefficients of the two materials $\left(P_{i j k}, \varepsilon_{i j}\right.$ and $\left.C_{i j k l}\right)$ are given in (40) and Table 2. In particular, the elasticity matrix $\left(C_{i j k l}\right)$ in terms of the Young's moduli $E_{1}, E_{2}, E_{3}$, the Poisson's ratios $v_{12}, v_{13}, v_{23}$ and the shear moduli $G_{12}, G_{13}, G_{23}$ of the material are shown. All the data displayed in Table 2 correspond exactly to two PZT ceramic materials used in [26]. The materials are orthotropic with constant elastic, piezoelectric and dielectric coefficients (cf. Tables VIII and XI in [26]).

Table 3

Solutions loc, $h_{0}$, mat, node and $d$ for the actuator optimization problem (mesh: $20 \times 20$ )

\begin{tabular}{|c|c|c|c|c|c|}
\hline$B C$ & $l o c=(i, j, p e)$ & $h_{0}$ & $m a t=\left(m a t_{1}, m a t_{2}\right)$ & Node & $d$ \\
\hline \multirow[t]{4}{*}{1} & $(1,4 \times 4,[4])$ & 0.00125 & (PZT-5, PZT-5A) & 441 & $1.248183 \mathrm{E}-05$ \\
\hline & $(2,4 \times 4,[4,5])$ & 0.00125 & (PZT-5, PZT-5A) & 441 & $2.293397 \mathrm{E}-05$ \\
\hline & $(3,4 \times 4,[3,4,5])$ & 0.00125 & (PZT-5, PZT-5A) & 441 & $3.348424 \mathrm{E}-05$ \\
\hline & $(4,4 \times 4,[3,4,5,9])$ & 0.00125 & (PZT-5, PZT-5A) & 441 & $4.029649 \mathrm{E}-05$ \\
\hline Fig. $4 \rightarrow$ & $(5,4 \times 4,[2,3,4,5,9])$ & 0.00125 & (PZT-5, PZT-5A) & 441 & $4.680007 \mathrm{E}-05$ \\
\hline \multirow[t]{4}{*}{2} & $(1,4 \times 4,[23])$ & 0 & (PZT-5, PZT-5A) & 431 & $4.818227 \mathrm{E}-06$ \\
\hline & $(2,4 \times 4,[17,23])$ & 0 & (PZT-5, PZT-5A) & 431 & $6.438335 \mathrm{E}-06$ \\
\hline & $(3,4 \times 4,[18,23,24])$ & 0 & (PZT-5, PZT-5A) & 432 & $8.721058 \mathrm{E}-06$ \\
\hline & $(4,4 \times 4,[18,19,23,24])$ & 0 & (PZT-5, PZT-5A) & 432 & $1.042312 \mathrm{E}-05$ \\
\hline Fig. $5 \rightarrow$ & $(5,4 \times 4,[17,18,19,22,23])$ & 0 & (PZT-5, PZT-5A) & 430 & $1.190036 \mathrm{E}-05$ \\
\hline \multirow[t]{4}{*}{3} & $(1,4 \times 4,[3])$ & 0 & (PZT-5, PZT-5A) & 11 & $4.828368 \mathrm{E}-06$ \\
\hline & $(2,4 \times 4,[2,3])$ & 0 & (PZT-5, PZT-5A) & 10 & $6.808695 \mathrm{E}-06$ \\
\hline & $(3,4 \times 4,[2,3,8])$ & 0 & (PZT-5, PZT-5A) & 10 & $8.708387 \mathrm{E}-06$ \\
\hline & $(4,4 \times 4,[2,3,7,8])$ & 0 & (PZT-5, PZT-5A) & 10 & $1.038399 \mathrm{E}-05$ \\
\hline Fig. $6 \rightarrow$ & $(5,4 \times 4,[2,3,7,8,9])$ & 0 & (PZT-5, PZT-5A) & 10 & $1.183562 \mathrm{E}-05$ \\
\hline \multirow[t]{4}{*}{4} & $(1,4 \times 4,[1])$ & 0.00125 & (PZT-5, PZT-5A) & 421 & $6.536013 \mathrm{E}-06$ \\
\hline & $(2,4 \times 4,[1,2])$ & 0.00125 & (PZT-5, PZT-5A) & 421 & $1.287488 \mathrm{E}-05$ \\
\hline & $(3,4 \times 4,[1,2,3])$ & 0.00125 & (PZT-5, PZT-5A) & 421 & $1.719750 \mathrm{E}-05$ \\
\hline & $(4,4 \times 4,[1,2,3,7])$ & 0.00125 & (PZT-5, PZT-5A) & 421 & $2.051966 \mathrm{E}-05$ \\
\hline Fig. $7 \rightarrow$ & $(5,4 \times 4,[1,2,3,6,7])$ & 0.00125 & (PZT-5, PZT-5A) & 421 & $2.366497 \mathrm{E}-05$ \\
\hline
\end{tabular}

Table 4

Solutions loc, $h_{0}$, mat, $e$ and elpot for the sensor optimization problem (mesh: $\left.20 \times 20\right)$

\begin{tabular}{|c|c|c|c|c|c|}
\hline$B C$ & $l o c=(i, j, p e)$ & $h_{0}$ & $m a t=m a t_{1}, m t_{2}$ & $e$ & elpot \\
\hline \multirow[t]{3}{*}{1} & $(1,4 \times 4,[21])$ & 0.00875 & (PZT-5, PZT-5A) & 5 & 0.894998 \\
\hline & $(2,4 \times 4,[21,22])$ & 0.00875 & (PZT-5, PZT-5A) & 5 & 1.654821 \\
\hline & $(3,4 \times 4,[16,21,22])$ & 0.00875 & (PZT-5, PZT-5A) & 5 & 2.380768 \\
\hline Fig. $8 \rightarrow$ & $(5,4 \times 4,[16,17,21,22,23])$ & 0.00875 & (PZT-5, PZT-5A) & 6 & 3.615470 \\
\hline \multirow[t]{2}{*}{2} & $(1,4 \times 4,[24])$ & 0.00875 & (PZT-5, PZT-5A) & 380 & 0.295855 \\
\hline & $(2,4 \times 4,[23,24])$ & 0.00875 & (PZT-5, PZT-5A) & 380 & 0.535390 \\
\hline Fig. $9 \rightarrow$ & $(5,4 \times 4,[19,22,23,24,25])$ & 0.00875 & (PZT-5, PZT-5A) & 380 & 0.903534 \\
\hline \multirow[t]{4}{*}{3} & $(1,4 \times 4,[23])$ & 0.00875 & (PZT-5, PZT-5A) & 380 & 0.239884 \\
\hline & $(2,4 \times 4,[23,24])$ & 0.00875 & (PZT-5, PZT-5A) & 380 & 0.535988 \\
\hline & $(3,4 \times 4,[23,24,25])$ & 0.00875 & (PZT-5, PZT-5A) & 380 & 0.677186 \\
\hline & $(4,4 \times 4,[22,23,24,25])$ & 0.00875 & (PZT-5, PZT-5A) & 380 & 0.793471 \\
\hline $\begin{array}{l}\text { Fig. } 10 \rightarrow \\
4\end{array}$ & $(5,4 \times 4,[19,22,23,24,25])$ & 0.00875 & (PZT-5, PZT-5A) & 380 & 0.904455 \\
\hline Fig. $11 \rightarrow$ & $(5,4 \times 4,[16,21,22,23,24]$ & 0.00875 & (PZT-5, PZT-5A) & 360 & 2.330120 \\
\hline
\end{tabular}



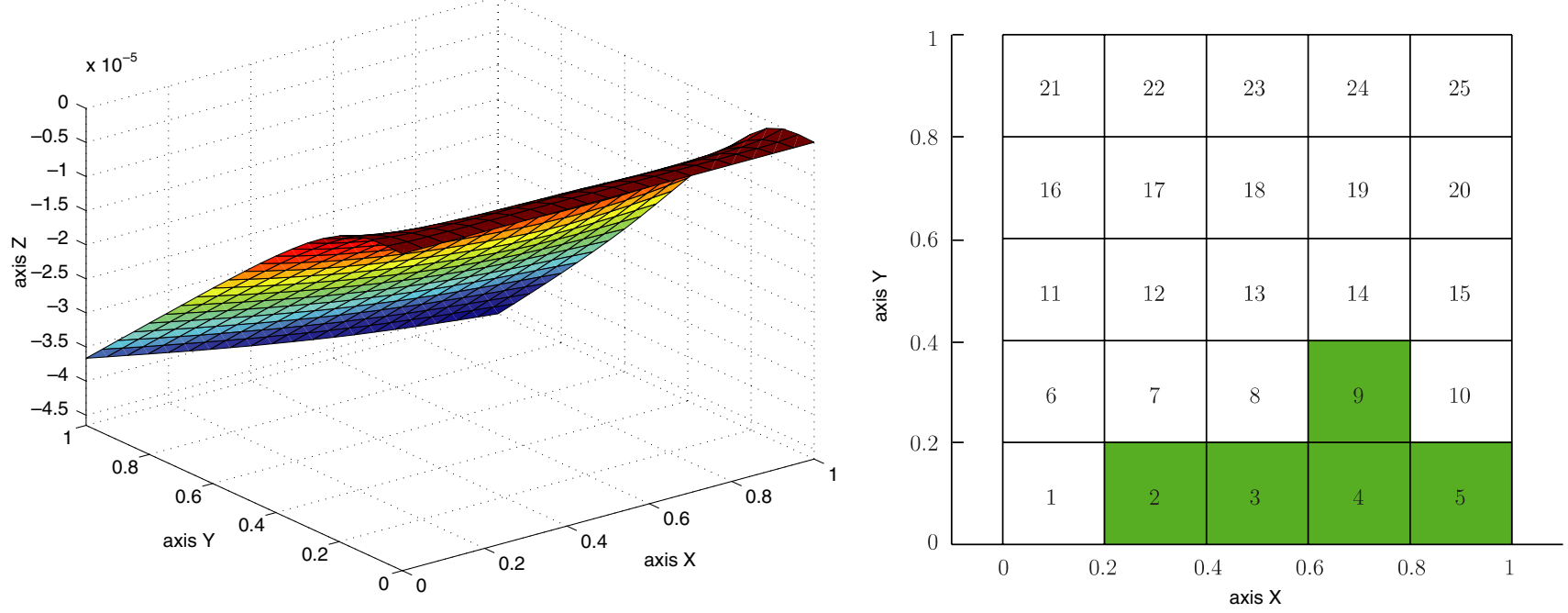

Fig. 4. Actuator optimization problem: transverse displacement of the plate's middle plane for $B C=1$ (left plot) and corresponding optimal position $p e=[2,3,4,5,9]$ of the regions where the non-zero electric potential difference is applied (right plot).
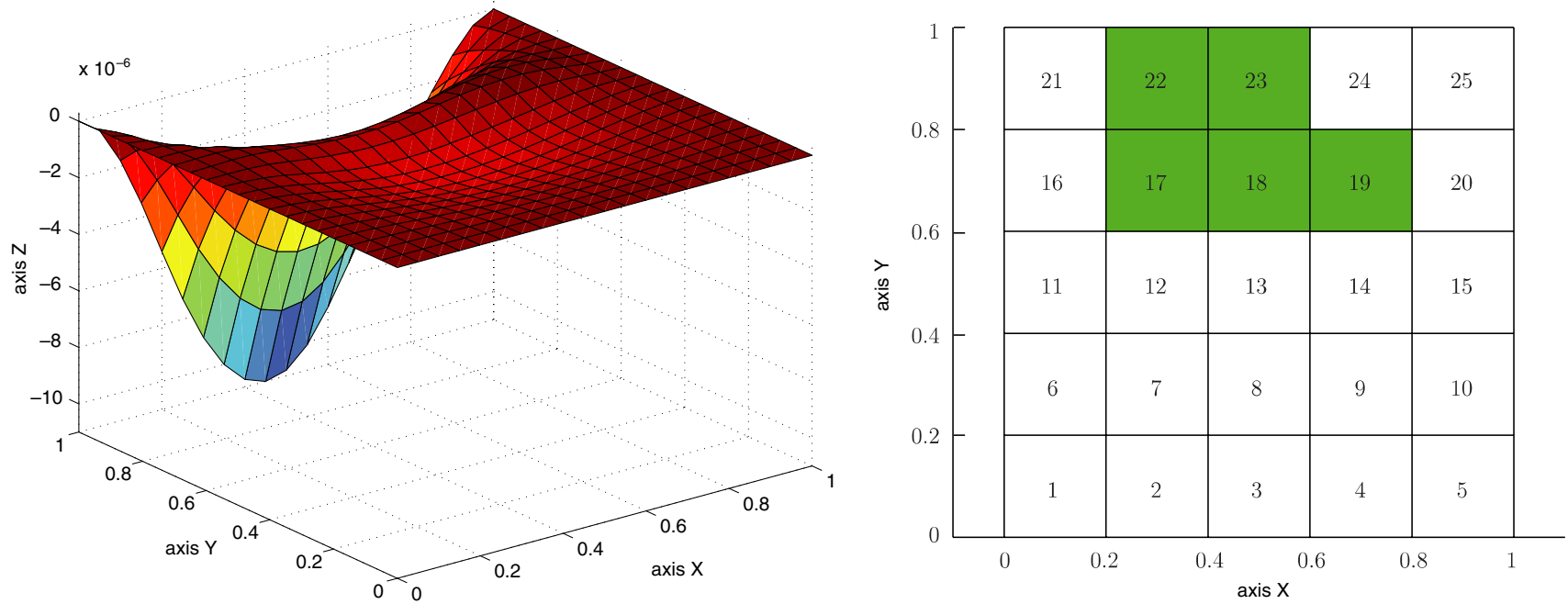

Fig. 5. Actuator optimization problem: transverse displacement of the plate's middle plane for $B C=2$ (left plot) and corresponding optimal position $p e=[17,18,19,22,23]$ of the regions where the non-zero electric potential difference is applied (right plot).
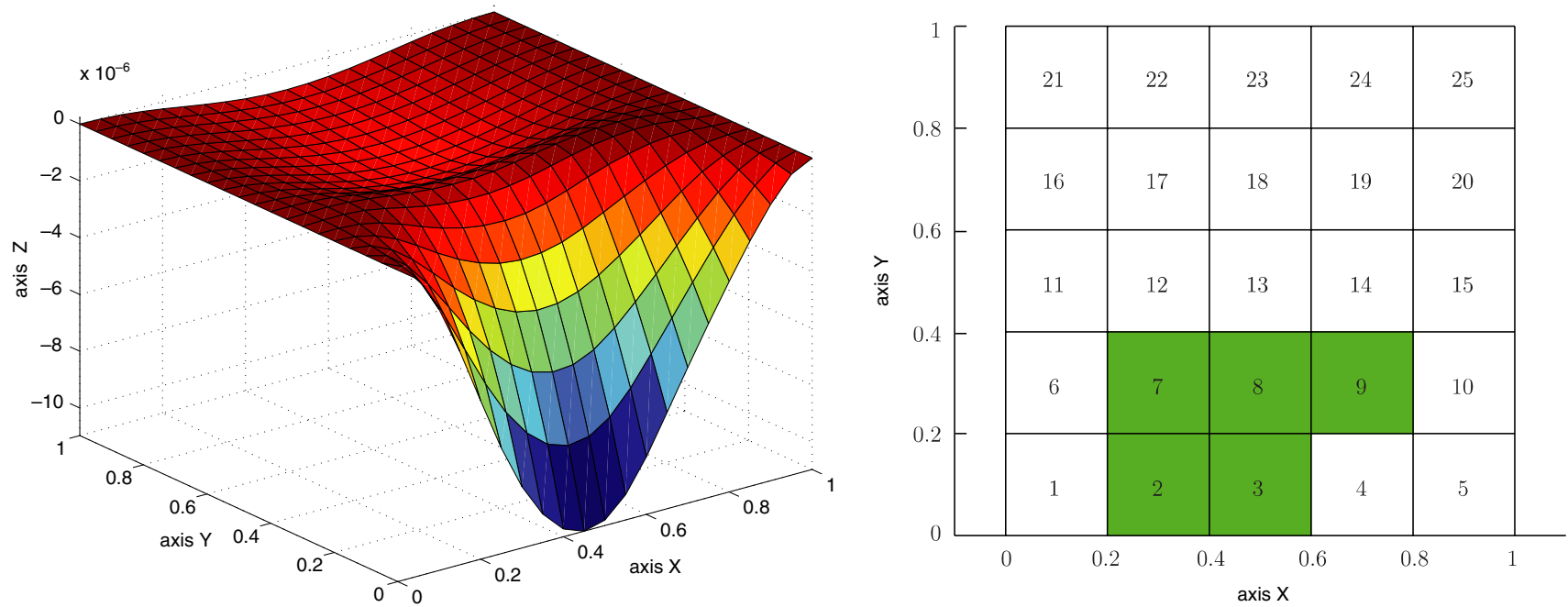

Fig. 6. Actuator optimization problem: transverse displacement of the plate's middle plane for $B C=3$ (left plot) and corresponding optimal position $p e=[2,3,7,8,9]$ of the regions where the non-zero electric potential difference is applied (right plot). 

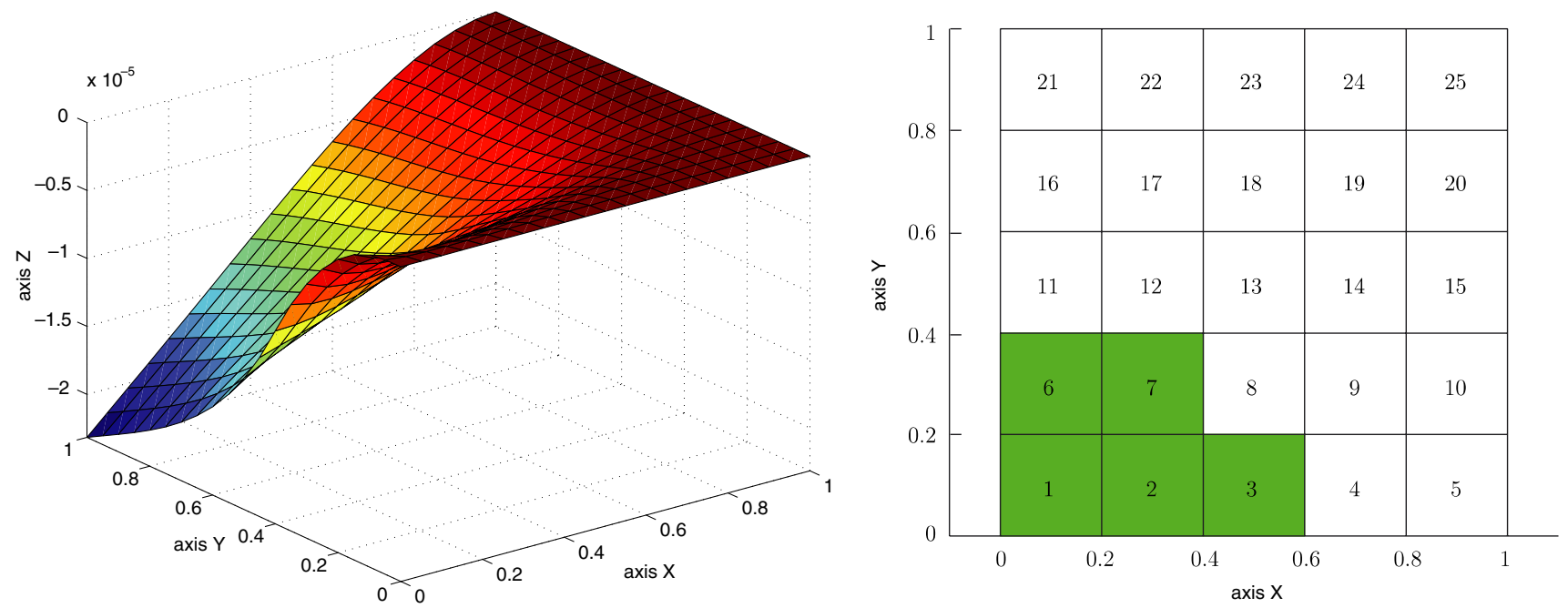

Fig. 7. Actuator optimization problem: transverse displacement of the plate's middle plane for $B C=4$ (left plot) and corresponding optimal position $p e=[1,2,3,6,7]$ of the regions where the non-zero electric potential difference is applied (right plot).
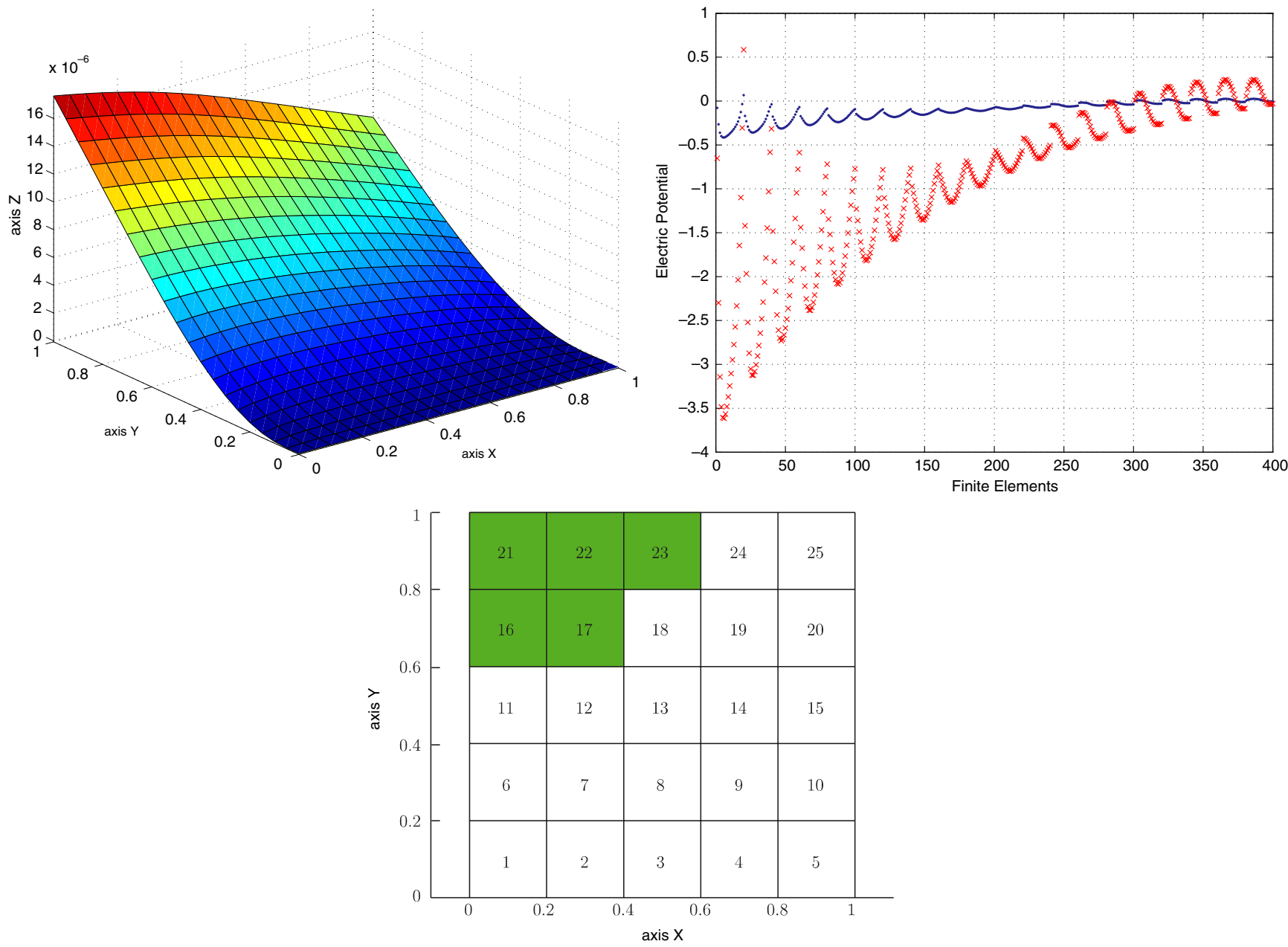

Fig. 8. Sensor optimization problem: transverse displacement of the plate's middle plane for $B C=1$ (upper left plot), electric potentials (cross mark $\varphi_{\left\{\omega^{e} \times\left\{z_{1}\right\}\right.}$ on lamina 1 and dotted $-\varphi_{\mid \omega^{e} \times\left\{z_{2}\right\}}$ on lamina 2) (upper right plot) and corresponding optimal position pe $=[16,17,21,22,23]$ of the regions where the non-zero mechanical forces are applied (lower plot). 


$$
\begin{aligned}
& {\left[\begin{array}{cccccc}
P_{111} & P_{122} & P_{133} & P_{123} & P_{131} & P_{112} \\
P_{211} & P_{222} & P_{233} & P_{223} & P_{231} & P_{212} \\
P_{311} & P_{322} & P_{333} & P_{323} & P_{331} & P_{312}
\end{array}\right]=\left[\begin{array}{cccccc}
0 & 0 & 0 & 0 & P_{15} & 0 \\
0 & 0 & 0 & 0 & 0 & P_{26} \\
P_{31} & P_{32} & P_{33} & 0 & 0 & 0
\end{array}\right]} \\
& {\left[\begin{array}{ccc}
\varepsilon_{11} & \varepsilon_{12} & \varepsilon_{13} \\
& \varepsilon_{22} & \varepsilon_{23} \\
\text { sym. } & & \varepsilon_{33}
\end{array}\right]=\varepsilon_{33}\left[\begin{array}{lll}
1 & 0 & 0 \\
0 & 1 & 0 \\
0 & 0 & 1
\end{array}\right]}
\end{aligned}
$$

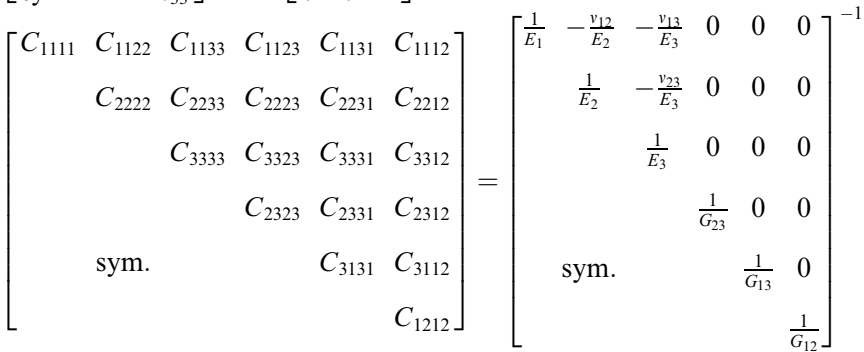

In Tables 1 and 2 , the unit symbols $\mathrm{m}, \mathrm{V}, \mathrm{N}, \mathrm{GPa}$, $\mathrm{C} \mathrm{m}^{-2}$ and $\mathrm{F} \mathrm{m}^{-1}$ mean, respectively, meter, volt, newton, giga pascal, coulomb per square meter and farad per meter.

\subsubsection{Genetic algorithms}

In general, engineering problems involve multiple conflicting objectives. For these problems no single solution that is optimal with respect to all objectives exists. Instead, there is a set of optimal solutions, known as Pareto optimal solutions, reflecting compromises between the objectives. Genetic algorithms (cf. [24]) are population based algorithms and, therefore, particularly suitable to tackle multiobjective problems. They can, in principle, find multiple widely different Pareto-optimal solutions in a single run (cf. [25]). Furthermore, they do not require any differentiability or convexity assumptions and can deal with complex search spaces, as well as non-convex Pareto fronts.

We apply the elitist genetic algorithm, described in [3] to the actuator and sensor multi-objective optimization problems. We note that the genetic algorithm used in this paper is also similar to the one applied in [4] for the analysis of the actuator effect of a single plate made of a transversely isotropic piezoelectric material. However, the mechanical model considered in the present paper is more complex
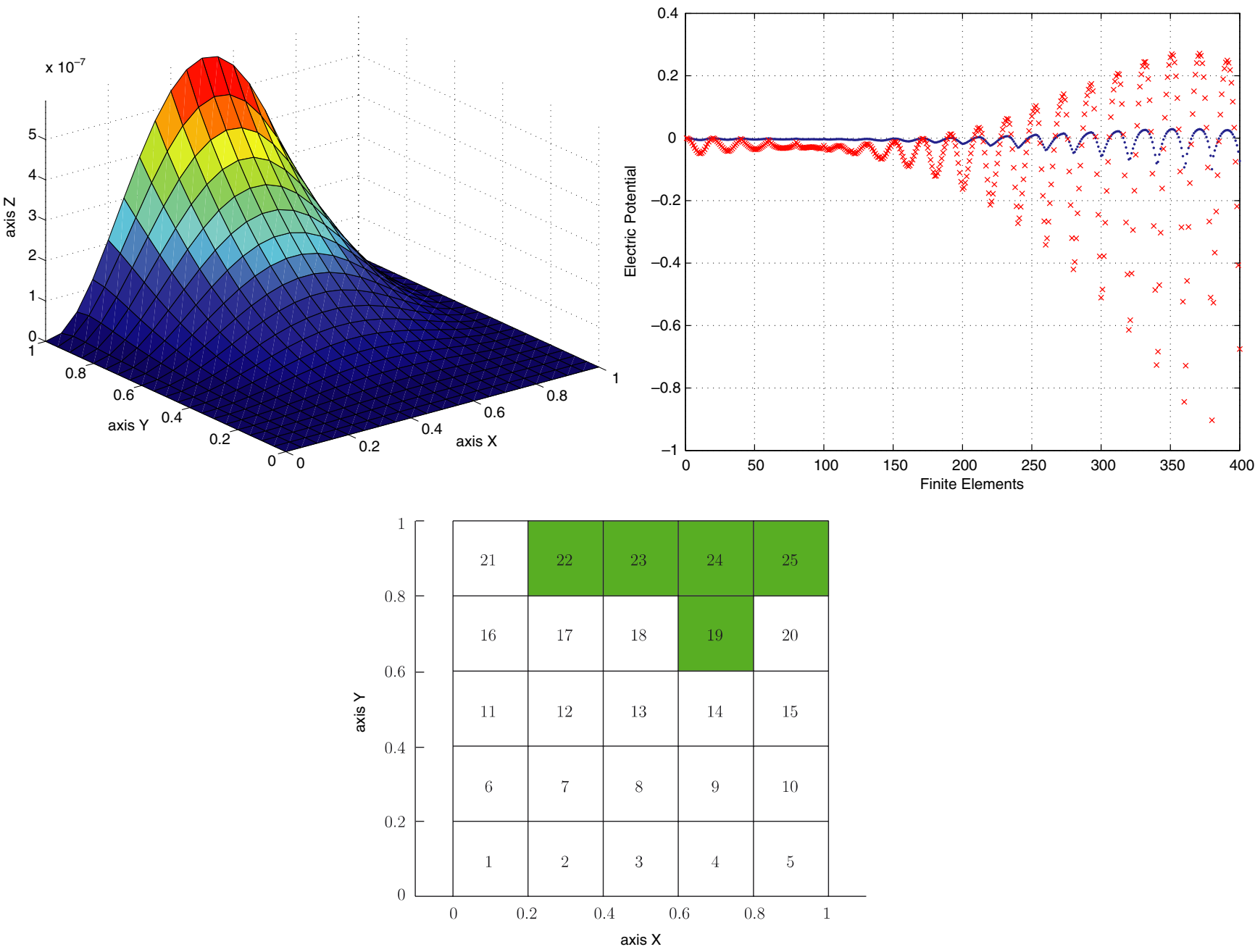

Fig. 9. Sensor optimization problem: transverse displacement of the plate's middle plane for $B C=2$ (upper left plot), electric potentials (cross mark $\varphi_{\mid \omega^{e} \times\left\{z_{1}\right\}}$ on lamina 1 and dotted $-\varphi_{\mid \omega^{e} \times\left\{z_{2}\right\}}$ on lamina 2 ) (upper right plot), and corresponding optimal position $p e=[19,22,23,24,25]$ of the regions where the non-zero mechanical forces are applied (lower plot). 
than the one in [4]. In fact, in the present model, the plate is laminated and made of different materials and therefore the tangential and transverse mechanical displacements are coupled (this did not occur in [4]). Moreover we deal with additional optimization variables related to the thicknesses of the layers and the order of the materials. We discuss both the actuator and sensor effects.

We now shortly describe some technical features and the parameters of this genetic algorithm. For both problems (37) and (38), the optimization variables $l o c=(i, j, p e), t$ and mat are encoded using binary strings (referred also as chromosomes) with a total length of 30 bits. The first 25 bits represent the sequence of the 25 regions: 1 means that a non-zero electric potential difference or a non-zero mechanical load is applied in this region, while 0 means that the applied electric potential difference or the mechanical load is equal to zero. Since only two materials are considered, the next bit suffices to represent the order of the materials: 1 represents the material vector $m a t=\left(m_{a t}\right.$, $\left.m a t_{2}\right)$, while 0 corresponds to $m a t=\left(m_{a} t_{2}, m_{1} t_{1}\right)$. The remaining 4 bits of the binary string represent the parameter $h_{0} \in \mathbb{R}$ (related to the thicknesses of the layers, cf. (39)) as a small constant ranging from $-\frac{7 h}{8}$ to $\frac{7 h}{8}$, allowing 16 values for $h_{0}$.

For the actuator problem, to each string we assign a displacement $u$, which is the solution of the inner linear system $K u=F$ in problem (37). For the sensor problem, to each chromosome we assign the vector of the electrical potentials $\varphi_{\mid \omega^{e} \times\left\{z_{s}\right\}}$ with $s=1,2$, and $e=1, \ldots, m$, where $m$ is the total number of finite elements.

The genetic algorithms is stopped after 100 generations. In all numerical tests we use an initial population size of 100 chromosomes. A tournament selection, a two point crossover and a uniform mutation are adopted. The crossover probability is 0.7 . The mutation probability is given by $\frac{1}{b}$, where $b$ is the binary string length, that is $b=30$. The elitism level considered is 10 . The value of sigma share $\left(\sigma_{\text {share }}\right)$ is taken equal to 1 . For sharing purposes, the dis-
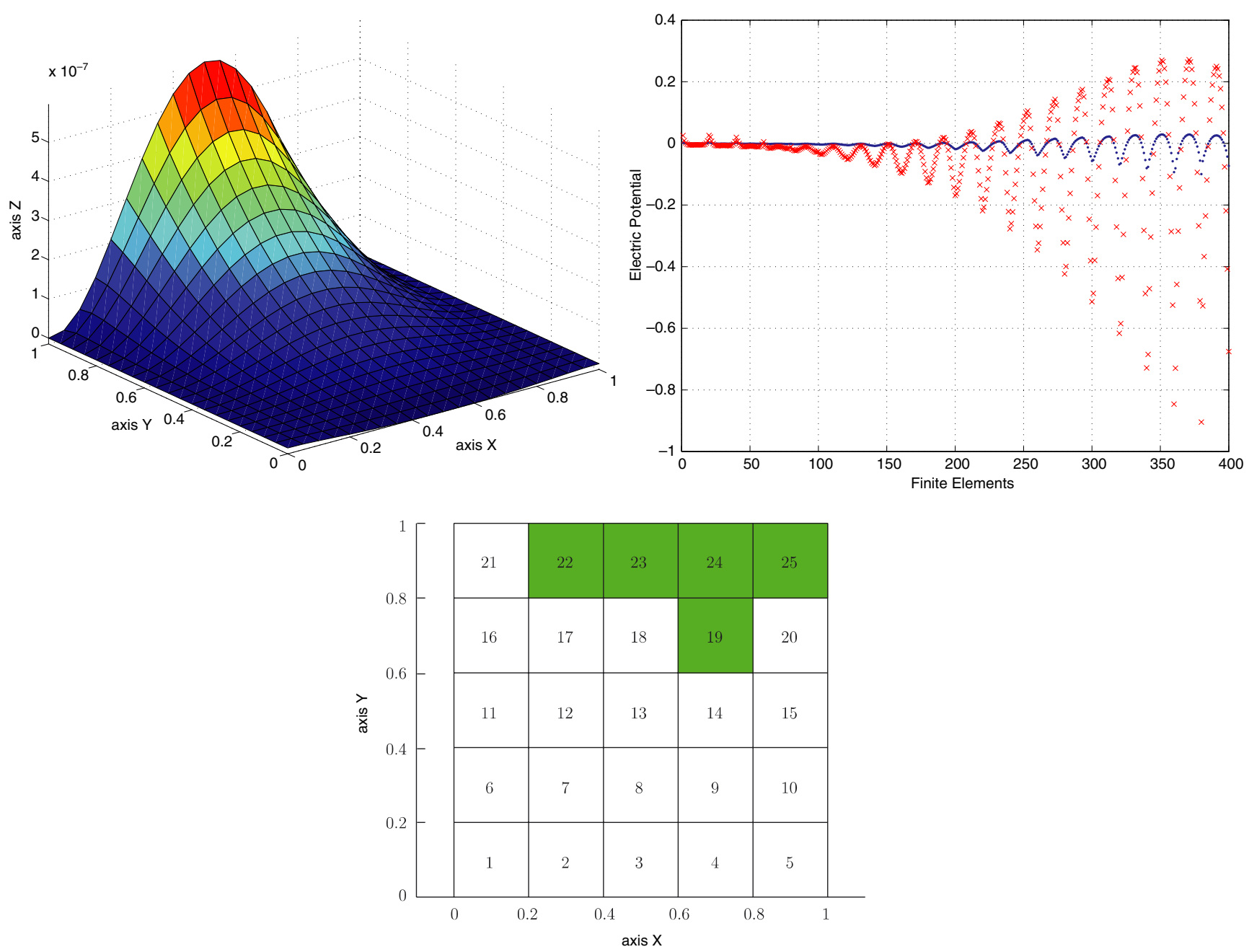

Fig. 10. Sensor optimization problem: transverse displacement of the plate's middle plane for $B C=3$ (upper left plot), electric potentials (cross mark $\varphi_{\left\{\omega^{e} \times\left\{z_{1}\right\}\right.}$ on lamina 1 , and, dotted $-\varphi_{\mid \omega^{e} \times\left\{z_{2}\right\}}$ on lamina 2) (upper right plot) and corresponding optimal position $p e=[19,22,23,24,25]$ of the regions where the non-zero mechanical forces are applied (lower plot). 
tance measure considered is the Hamming distance between chromosomes (cf. [24]).

\subsubsection{Solutions}

For all our tests, the stiffness matrices $K$ and force vectors $F$ have been evaluated with the subroutines planre and platre of the CALFEM toolbox of MATLAB [27]. The genetic algorithms have been implemented in $\mathrm{C}++$.

The Fig. 2 shows the objective values $d$ of the Pareto optimal solutions for the actuator multi-objective problem (37) as a function of the number $i$ of regions. We observe an increase of the displacement $d$ with the number of regions $i$, but for some values of $i$ there are not Pareto optimal solutions. This happens for $23 \leqslant i \leqslant 25$ if $B C=1$, for $22 \leqslant i \leqslant 25$ if $B C=2$, for $20 \leqslant i \leqslant 25$ if $B C=3$, and for $19 \leqslant i \leqslant 25$ if $B C=4$. This means, for example for the latter case $B C=4$, that to achieve a maximum displacement $d$ it suffices to apply the electric potential difference to 18 regions, because the application of a non-zero electric potential difference in more than 18 regions (in 21 or 23 , for example) will not increase the maximum displacement value $d$.

Analogously, Fig. 3 represents the objective values elpot of the Pareto optimal solutions for the sensor multi-objective problem (38) as a function of the number $i$ of regions, where mechanical forces are applied.

We observe the same phenomena as in Fig. 2. In general, the objective value elpot increases with the number $i$, but for some $i$ there are not Pareto optimal solutions. Namely, for $24 \leqslant i \leqslant 25$ if $B C=1$, for $23 \leqslant i \leqslant 25$ if $B C=2$, for $18 \leqslant i \leqslant 25$ if $B C=3$, and for $22 \leqslant i \leqslant 25$ if $B C=4$.

Some of the Pareto optimal solutions produced by the genetic algorithms are also displayed in Table 3 (for the actuator optimization problem) and Table 4 (for the sensor optimization problem).

In Table 3, node represents the number of the node, in which the maximum displacement $d$ is attained. The Figs.
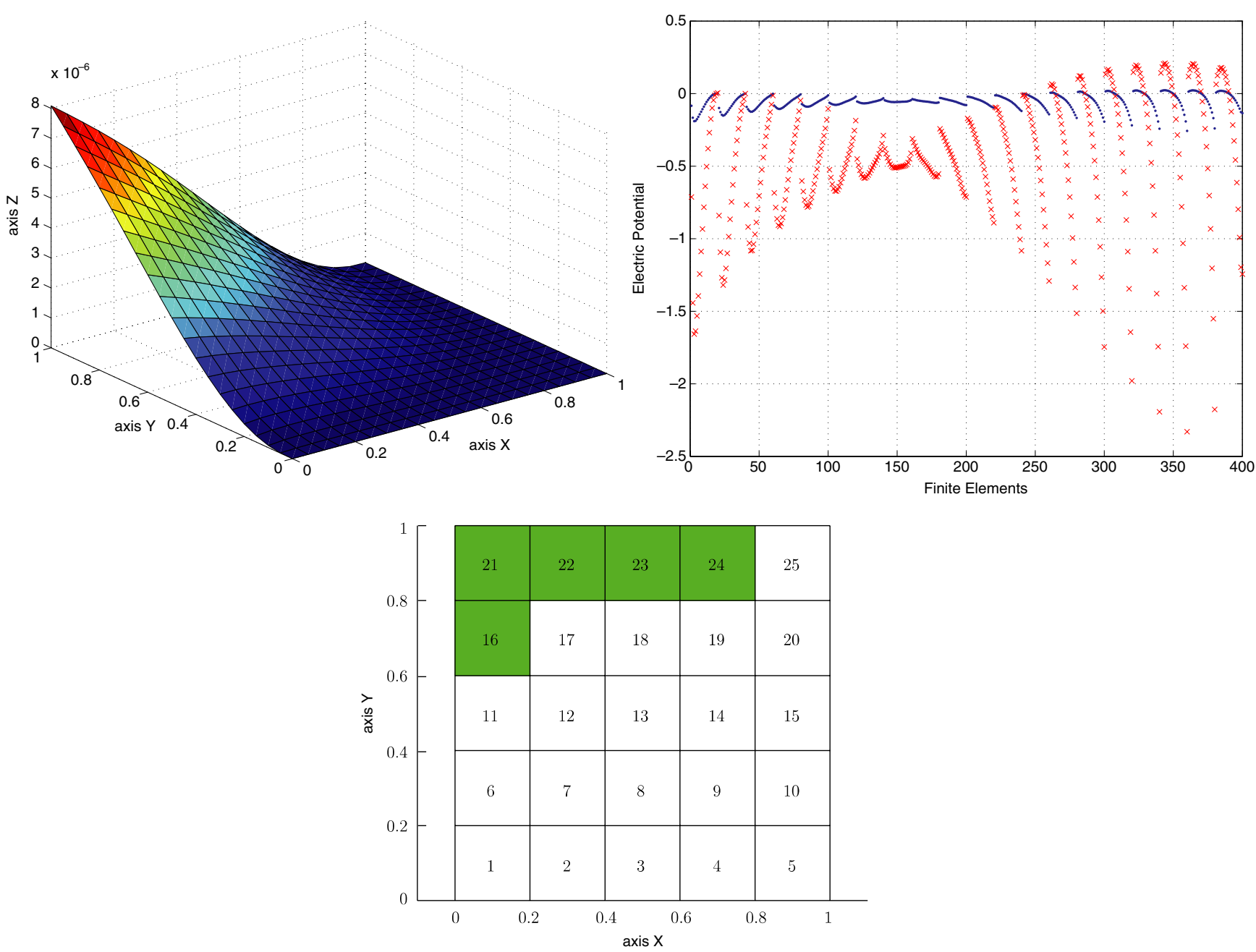

Fig. 11. Sensor optimization problem: transverse displacement of the plate's middle plane for $B C=4$ (upper left plot), electric potentials (cross mark $\varphi_{\mid \omega^{e} \times\left\{z_{1}\right\}}$ on lamina 1 , and, dotted $-\varphi_{\mid \omega^{e} \times\left\{z_{2}\right\}}$ on lamina 2) (upper right plot) and corresponding optimal position $p e=[16,21,22,23,24]$ of the regions where the non-zero mechanical forces are applied (lower plot). 
4-7 (labelling four rows in Table 3) represent the plots of the transverse displacements of the plate's middle plane for the corresponding $B C, l o c$ and mat.

In Table $4, e$ is the number of the finite element where the maximum electric potential elpot is attained for the sensor optimization problem. The Figs. 8-11 (labelling four rows in Table 4) depict the transverse displacement of the plate's middle plane and plot the electric potentials measured at the middle plane of each lamina and at each finite element for the indicated four groups of $B C, l o c$ and mat.

In Tables 3 and 4, we have omitted all the symmetric solutions loc, $h_{0}$ and mat producing the same objective values $d$ and elpot. In fact, due to the symmetry of the plate and the boundary conditions, there are always several locations pe and symmetrical values of $h_{0}$ and mat that lead to the same $d$ and elpot.

Finally we have also tested the influence of the refinement of the finite element mesh in the numerical results produced by the genetic algorithms. We have done experiments with three meshes with $5 \times 5,10 \times 10$ and $20 \times 20$ finite elements, which means that the variable $j$ in $l o c$ becomes $j=1$ for the $5 \times 5$ mesh, $j=4=2 \times 2$ for the $10 \times 10$ mesh and $j=16=4 \times 4$ for the $20 \times 20$ mesh. For these three different discretizations we observe a similar behavior of the objective values $d$, elpot and $h_{0}$, mat, as well as a similar location $p e$ for the optimal regions.

\section{Conclusions}

In this paper, we have developed a piezoelectric model for a thin plate made of a completely anisotropic material. For the sake of validating the model, a laminated plate with two piezoelectric materials of variable thickness is used. For this plate, the actuator and the sensor effects are studied using bi-objective optimizations problems. Due to their characteristics (non-differentiability and non-convexity), genetic algorithms are used to obtain (Pareto-optimal) solutions. For the actuator optimization problem the objectives are to maximize the mechanical displacement while, at the same time, minimize the number of regions where a non-zero electric potential is applied. For the sensor effect, the objectives are the maximization of the electric potential inside the plate while minimizating the number of regions which are subject to mechanical loads. For various boundary conditions we show where to place the applied electric potentials or the mechanical loads, taking into consideration the thickness and the order of the materials. Future work will aim at solving problems with more involved optimization variables and new objectives (e.g., to obtain a pre-defined mechanical deformation of the plate) using genetic algorithms. Moreover, we also intend to apply techniques from continuous optimization such as optimal control for the investigation and the design of smart materials involving piezoelectric plates.

\section{Acknowledgements}

This work is partially supported by the project Mathematical analysis of piezoelectric problems (FCT-POCI/ MAT/59502/2004 of Portugal), and is part of the project New materials, adaptive systems and their nonlinearities; modelling, control and numerical simulation (European Community program HRN-CT-2002-00284).

\section{References}

[1] Ikeda T. Fundamentals of piezoelectricity. New York: Oxford University Press; 1990.

[2] Smith R. Smart material systems: model development (Frontiers in applied mathematics; 32). Philadelphia: SIAM; 2005.

[3] Costa L, Oliveira P. An elitist genetic algorithm for multiobjective optimization. In: Resende MGC, de Sousa JP, editors. Metaheuristics: computer decision-making. Kluwer Academic Publishers; 2003. p. 217-36.

[4] Costa L, Oliveira P, Figueiredo IN, Leal R. Actuator effect of a piezoelectric anisotropic plate model. Mech Adv Mater Struct 2006;13(5):403-17.

[5] Ciarlet PG, Destuynder P. A justification of the two-dimensional plate model. J Méc 1979;18:315-44.

[6] Ciarlet PG. In: Mathematical elasticity. Theory of plates, Vol. II. Amsterdam: North-Holland; 1997.

[7] Maugin GA, Attou D. An asymptotic theory of thin piezoelectric plates. Quart J Mech Appl Math 1990;43:347-62.

[8] Rahmoune M, Benjeddou A, Ohayon R. New thin piezoelectric plate models. J Intell Mater Syst Struct 1998;9:1017-29.

[9] Sene A. Modelling of piezoelectric static thin plates. Asymptot Anal 2001;25(1):1-20.

[10] Raoult A, Sene A. Modelling of piezoelectric plates including magnetic effects. Asymptot Anal 2003;34(1):1-40.

[11] Figueiredo IN, Leal CF. A piezoelectric anisotropic plate model. Asymptot Anal 2005;44(3-4):327-46.

[12] Collard C, Miara B. Two-dimensional models for geometrically nonlinear thin piezoelectric shells. Asymptot Anal 2003;31(2) 113-51.

[13] Bernadou M, Haenel C. Modelization and numerical approximation of piezoelectric thin shells. I. The continuous problems. Comput Methods Appl Mech Eng 2003;192(37-38):4003-43.

[14] Bernadou M, Haenel C. Modelization and numerical approximation of piezoelectric thin shells. II. Approximation by finite element methods and numerical experiments. Comput Methods Appl Mech Eng 2003;192(37-38):4045-73.

[15] Bernadou M, Haenel C. Modelization and numerical approximation of piezoelectric thin shells. III. From the patches to the active structures. Comput Methods Appl Mech Eng 2003;192(37-38): 4075-107.

[16] Reddy JN. On laminated composite plates with integrated sensors and actuators. Eng Struct 1999;21:568-93.

[17] Geis W, Sändig A-M, Mishuris G. Piezoelectricity in multi-layer actuators: modelling and analysis in two and three-dimensions, Preprint IANS, 2003/23, Univ. Stuttgart, Germany, 2003.

[18] Geis W, Mishuris G, Sändig A-M. Asymptotic models for piezoelectric stack actuators with thin metal inclusions, Preprint IANS, 2004/ 01, Univ. Stuttgart, Germany, 2004.

[19] Geis W. Numerical simulation of linear models for piezoelectric stack actuators, Preprint IANS, 2005/07, Univ. Stuttgart, Germany, 2005.

[20] Halim Dunant, Reza Moheimani SO. An optimization approach to optimal placement of collocated piezoelectric actuators and sensors on a thin plate. Mechatronics 2003;13:27-47.

[21] Caruso G, Galeani S, Menini L. On actuators/sensors placement for collocated flexible plates. In: Proceedings of the 11th IEEE Mediter- 
ranean on control and automation, (Rhodes-Greece), 18-20 June, 2003.

[22] Sadri AM, Wright JR, Wynne RJ. Modelling and optimal placement of piezoelectric actuators in isotropic plates using genetic algorithms. Smart Mater Struct 1999;8:490-8.

[23] Ciarlet PG. The finite element method for elliptic problems. Amsterdam: North-Holland; 1978.

[24] Goldberg D. Genetic algorithms in search, optimization and machine learning. Reading, MA: Addison-Wesley; 1989.
[25] Deb K. Multi-objective optimization using evolutionary algorithms. England: John Wiley \& Sons; 2001.

[26] Klinkel S, Wagner W. A geometrically nonlinear piezoelectric solid shell element based on a mixed multi-field variational formulation. Int J Numer Methods Eng 2006;65(3):349-82.

[27] CALFEM. A finite element toolbox to MATLAB, Version 3.4, Structural Mechanics and Solid Mechanics, Department of Mechanics and Materials, Lund University, Sweden, 2004. <http:// www.byggmek.lth.se/CALFEM $>$. 